Short title: soapberry saponin biosynthesis mechanism

\title{
Metabolome and transcriptome analysis reveals components regulating triterpenoid saponin biosynthesis of soapberry
} Yuanyuan $\mathrm{Xu}^{1,2,3, \dagger}$, Guochun Zhao ${ }^{1,2,3, \dagger}$, Xiangqin $\mathrm{Ji}^{4}$, Jiming Liu ${ }^{1,2,3}$, Tianyun Zhao ${ }^{1,2,3}$, Yuan $\mathrm{Gao}^{5}$, Shilun Gao ${ }^{1,2,3}$, Yingying Hao ${ }^{1,2,3}$, Yuhan Gao ${ }^{1,2,3}$, Lixian Wang ${ }^{1,2,3}$, Xuehuang Weng ${ }^{6}$, Zhong Chen ${ }^{1,2,3,7, *}$, Liming Jia ${ }^{1,2,3, *}$

${ }^{1}$ Key Laboratory of Silviculture and Conservation of the Ministry of Education, College of Forestry, Beijing Forestry University, Beijing 100083, China

${ }^{2}$ National Energy R\&D Center for Non-food Biomass, Beijing Forestry University, Beijing 100083, China

${ }^{3}$ National Innovation Alliance of Sapindus Industry, Beijing Forestry University, Beijing 100083, China

${ }^{4}$ Hangzhou KaiTai Biotechnology Co., Ltd, Hangzhou, Zhejiang 310030, China

${ }^{5}$ Planning and Design Institute of Forest Products Industry, National Forestry and Grassland Administration, Beijing 100010, China

${ }^{6}$ Yuanhua Forestry Biological Technology Co., Ltd., Sanming, Fujian 354500, China

${ }^{7}$ Beijing Advanced Innovation Center for Tree Breeding by Molecular Design, Beijing Forestry University, Beijing 100083, China

ORCID IDs: 0000-0003-2727-8929 (Y.X.); 0000-0001-8362-6188 (G.Z.);

0000-0003-3196-5238 (Z.C.); 0000-0002-6680-041X (L.J.)

${ }^{\dagger}$ These authors contributed equally to this work.

*Authors for correspondence: Zhong Chen (zhongchen@bjfu.edu.cn) and Liming Jia (jlm@bjfu.edu.cn)

College of Forestry, Beijing Forestry University, 35 E Qinghua Road, Beijing 100083, China Tel.: +86-13020086124 (Z. Chen); +86-13501103773 (L. Jia) 
The authors responsible for distribution of materials integral to the findings presented in this article in accordance with the policy described in the Instructions for Authors (https://academic.oup.com/plphys/pages/General-Instructions) are Zhong Chen (zhongchen@bjfu.edu.cn) and Liming Jia (jlm@bjfu.edu.cn).

One-sentence summary: Combining metabolome and transcriptome analysis to identify saponin kinetic patterns, gene networks, and key candidate genes and transcription factors involved in saponin biosynthesis of soapberry.

Author contributions: L.J. and Z.C. designed research; Y.X., S.G., L.W., and X.W. collected plant material; Y.X., G.Z., and Y.H. performed experiments; X.J., J.L., and Y.G. analyzed data; Y.X. and Z.C. wrote the manuscript. T.Z. and Y.H.G. modified the language and revised the manuscript. All authors contributed to the article and approved the final manuscript. Y.X. and G.Z. contributed equally to this work.

\section{Abstract}

Soapberry (Sapindus mukorossi Gaertn.) pericarps are rich in valuable bioactive triterpenoid saponins. However, the saponin content dynamics and the molecular regulatory network of saponin biosynthesis in soapberry pericarps remain largely unclear. Here, we performed combined metabolite profiling and transcriptome analysis to identify saponin accumulation kinetic patterns, investigate gene networks, and characterize key candidate genes and transcription factors involved in saponin biosynthesis in soapberry pericarps. A total of 54 saponins were tentatively identified, including 25 that were differentially accumulated. Furthermore, 49 genes putatively involved in sapogenin backbone biosynthesis and some candidate genes assumed to be responsible for the backbone modification, including 41 cytochrome P450s and 45 glycosyltransferases, were identified. Saponin-specific clusters/modules were identified by Mfuzz clustering and weighted gene co-expression network analysis, and one $\mathrm{TF}-$ gene regulatory network underlying saponin biosynthesis was proposed. The results of yeast one-hybrid assay and electrophoretic mobility shift assay suggested that SmbHLH2, SmTCP4, and SmWRKY27 may play important roles in the triterpenoid saponin 
biosynthesis by directly regulating the transcription of $S m C Y P 71 D-3$ in soapberry pericarp. Overall, these findings provide valuable information for understanding the molecular regulatory mechanism of saponin biosynthesis, enriching the gene resources, and guiding further research on triterpenoid saponin accumulation in soapberry pericarps.

Keywords: Sapindus mukorossi, metabolome, transcriptome, triterpenoid saponin, biosynthesis, transcription factor

\section{Introduction}

Soapberry (Sapindus mukorossi Gaertn.) is an economically important tree belonging to the Sapindaceae that grows in southern China, the Indochina Peninsula, India, Japan and North Korea (Jia and Sun, 2012; Sun et al., 2017). The tree has been used in traditional medicine for centuries in China because its pericarps possess many pharmacological properties, including triterpenoid saponins with excellent antibacterial, antitumor, hepatoprotective, antihyperglycemic, antidyslipidemic, and insecticidal activities (Li, 1975; Upadhyay and Singh, 2012; Xu et al., 2018). In addition, soapberry pericarp has been traditionally used as a detergent because of its widespread ability to form stable soap-like foams in aqueous solution (Li, 1975; Upadhyay and Singh, 2012). To date, more than 70 triterpenoid saponin compounds have been identified in soapberry, which are mainly divided into four structural types, namely, oleanane, lupine, dammarane, and tirucullane types (Upadhyay and Singh, 2012; Xu et al., 2018). The majority of studies on soapberry saponins have focused on constituents and bioactivity, while there have been no reports regarding the biosynthesis of these physiologically active substances.

The biosynthesis of plant triterpenoid saponins is a complex process, and the whole pathway can be divided into three stages: isopentenyl diphosphate (IPP) and its isomer, dimethylallyl diphosphate (DMAPP), derived either from condensation of acetyl-CoA in the cytosolic mevalonic acid (MVA) pathway or from pyruvate and phosphoglyceraldehyde in the plastidial 2-C-methyl-D-erythritol 4-phosphate (MEP) pathway; IPP and DMAPP are catalyzed by the corresponding enzymes to produce 2,3-oxidosqualene; and 2,3-oxidosqualene is cyclized to a triterpenoid backbone by oxidosqualene cyclases (OSCs). These molecules are then 
chemically modified by cytochrome P450 monooxygenase (CYP450) and UDP-glycosyltransferase (UGT), resulting in the production of different types of triterpenoid saponins (Augustin et al., 2011; Yang et al., 2018; Zhao and Li, 2018; Xu et al., 2021). Several transcription factors (TFs) have been reported to be involved in the biosynthesis of triterpenoid saponins by regulating the expression of saponin biosynthetic genes, such as bHLH (Mertens et al., 2016), AP2/ERF (Deng et al., 2017), bZIP (Xu et al., 2019), and WRKY (Singh et al., 2017).

With the further development and improvement of multiomics technologies, the joint analysis of the metabolome and transcriptome has been widely conducted to determine the metabolic pathways and regulatory genes in numerous plant species, including Solanum lycopersicum (Li et al., 2020), Capsicum annuum (Liu et al., 2020), Colchicum spp., and Gloriosa spp. (Nett et al., 2020), Citrullus lanatus (Umer et al., 2020), Actinidia chinensis (Wang et al., 2021), and Zea mays (Zhang et al., 2021), as well as many trees, including Ginkgo biloba (Meng et al., 2019), Dimocarpus longan (Yi et al., 2020), and Pyrus spp. (Ni et al., 2020). However, there have been few studies involving integration of triterpenoid saponin metabolism and transcriptome data to explain the molecular regulatory mechanisms underpinning triterpenoid saponin biosynthesis in soapberry pericarps.

Here, we examined the triterpenoid saponin dynamics and the regulatory mechanisms underlying triterpenoid saponin biosynthesis in soapberry at eight pericarp growth stages. Through a combination of metabolite profiling and transcriptome analysis, saponin-specific clusters/modules were identified by Mfuzz clustering and weighted gene co-expression network analysis (WGCNA), and one TF-gene regulatory network controlling production and accumulation of triterpenoid saponin were constructed. In addition, we identified key TFs that modulate triterpenoid saponin biosynthesis metabolism by direct transcriptional regulation of their structural target genes. This study not only clarified the accumulation of saponins in soapberry pericarps during pericarp development, but also provided important insights into the regulation of triterpenoid saponin biosynthesis in soapberry. 


\section{Results}

\section{Identification of saponins in the soapberry pericarp at eight fruit growth stages}

To investigate the constituents and dynamic changes of saponins in the developing soapberry pericarp, the total saponin content of soapberry pericarp was measured at eight fruit growth stages (Figure 1A; Figure 1B). Total saponins showed rapid accumulated from S1 (12.30\%), moderate accumulated from S3 (19.04\%), peaked at S4 (19.30\%), and then decreased gradually, indicating that the saponin content changed dynamically during soapberry fruit growth. Metabolite profiling of pericarp was then performed at eight fruit growth stages by UHPLC-Q-Qrbitrap-MS. A total of 54 types of saponin (including 11 groups of isomers) were tentatively detected in all eight stages (Supplemental Table S1). Principal component analysis (PCA) confirmed that these saponins could be divided into two groups and exhibited clear separation among pericarp samples at different stages (Figure 1C). Based on their accumulation at different growth stages, the cluster dendrogram also distinguished two groups (Figure 1D), which was consistent with the PCA results. The saponins in cluster I $(n=9)$ accumulated preferentially at stage $\mathrm{S} 1$. Saponins in cluster II $(\mathrm{n}=45)$ accumulated mainly at stage S2, S4, S5, or S8 (Figure 1D). These results suggested that the saponin profile shows a dynamically changing pattern during pericarp growth. In addition, saponins 19, 20, 22, 25, and 31 were the major saponins and accumulated specifically at stage S4. Saponins 11 and 12 were also the major saponins and accumulated preferentially at stage S8 (Supplemental Table S1; Figure 1D).

Based on the pairwise comparison with $\mid \log _{2}$ (fold change) $\mid>1, p<0.05$, and VIP (variable importance in project) $>1$ as thresholds, a total of 25 differentially accumulated saponins (DASs) were identified (Supplemental Table S2). The 25 DASs were divided into three groups by Mfuzz clustering based on their accumulation levels (Ni et al., 2020) (Figure 1E; Supplemental Table S3). In cluster A, the saponins showed high accumulation at stage S1 or S2 and tapered off from stage S3 to S8. The content of saponins in cluster B increased continuously with fruit growth. For cluster $\mathrm{C}$, the levels of saponin accumulation showed an uptrend trend, had high levels at stages S2, S4, and S5, and then decreased from S6 (Figure 1E). 


\section{Transcriptome profiles of soapberry at eight growth stages}

After removal of low-quality reads, ambiguous reads, and adapter sequences, each library had 31.97-86.81 M clean reads (Supplemental Table S4). These clean reads were mapped to the soapberry reference genome with average match ratios of $87.87 \%$, and 23,783 genes predicted from the genome were found to be expressed in at least one sample with fragments per kilobase of transcript per million fragments mapped $($ FPKM) $>0$. Furthermore, 9114 novel genes were also identified, which were not included in the reference genome. Twenty randomly selected genes were verified by qRT-PCR, which suggested that the relative expression levels were basically consistent with the RNA-Seq data (Supplemental Figure S1), thus supporting the accuracy and reliability of the transcriptomic profiling results. Through differential expression analysis between eight pericarp growth stages, a total of 22,002 DEGs were identified, and the DEG numbers increased gradually with fruit growth (Supplemental Figure S2). In addition, the number of downregulated genes was greater than that of upregulated genes (Supplemental Figure S2), suggesting that downregulated genes may play more important roles in soapberry saponin biosynthesis. GO enrichment analysis of these DEGs indicated that they were widely distributed in three functional groups. The genes related to protein phosphorylation, transcription, DNA-templated, and carbohydrate metabolic process were predominant in the biological process category. In the cellular component category, the genes were mostly involved in endoplasmic reticulum membrane, vacuolar membrane, and vacuole. In the molecular function category, heme binding was predominant followed by iron ion binding and hydrolase activity (Supplemental Figure S3).

Subsequently, the 22,002 DEGs were grouped into 12 clusters by Mfuzz clustering based on their expression levels (Supplemental Figure S4). Among them, the expression of clusters 2, 9, and 11 showed consistency with the accumulation patterns of DASs (clusters A, B, and C). DEGs from cluster 2 (2442 DEGs) showed similar expression tendencies, and their expression levels were highest at stage S1 and then decreased with fruit growth. Conversely, in cluster 11, the expression profiles of most DEGs (3816) were the opposite of those in cluster 2, showing an uptrend over time and peaking at stage S8. The DEGs (1560) in cluster 9 generally shared a trend of first increasing and then decreasing. Kyoto Encyclopedia of Genes and Genomes (KEGG) 
pathway enrichment analysis revealed that some of the DEGs in these three clusters were related to terpenoid backbone biosynthesis and sesquiterpenoid and triterpenoid biosynthesis (Supplemental Figure S5), suggesting that these DEGs play key roles in saponin biosynthesis in soapberry.

\section{Expression patterns of saponin biosynthesis-related genes in soapberry pericarp}

To further explore the mechanism of saponin accumulation in soapberry pericarp, the expression patterns of genes possibly related to triterpenoid saponin biosynthesis were analyzed. In this study, we found 135 genes encoding the enzymes involved in the MVA pathway, MEP pathway, and saponin biosynthesis pathway (Figure 2; Supplemental Table S5). Among them, 49 genes were involved in putative backbone biosynthesis and some candidate genes were assumed to be responsible for backbone modification, including 41 CYP450s and 45 UGTs. In addition, phylogenetic analysis showed that most of the OSCs, CYP450s, and UGTs in our study had high degrees of identity with known examples (Supplemental Figure S6-S8). Most of the 135 candidate genes, such as 3-hydroxy-3-methylglutaryl-CoA reductase (HMGR), phosphomevalonate kinase (PMK), metholvalic-5-diphosphate decarboxylase (MVD), 4-cytidine diphosphate-2-C-methyl-D-erythritol kinase (CMK), 2-C-methyl-D-erythritol-2,4-cyclic phosphate synthetase (MDS), IPP isomerase (IDI), geranyl pyrophosphate synthetase (GPS), farnesyl pyrophosphate synthetase (FPS), squalene synthase (SS), squalene epoxidase (SE), $\beta$-amyrin synthase ( $\beta$-AS), CYP450 and UGT, were highly expressed at the early stages of pericarp growth (stages S1-S4) (Figure 2). This was consistent with the trend that the contents of total saponins and most saponins were higher in the early pericarp growth stages (such as stages S2 and S4), indicating that saponins were mainly synthesized in the early stages of pericarp growth and we can further screen for genes closely related to saponin biosynthesis from these genes.

\section{Expression patterns of TFs in soapberry pericarp}

A total of 1785 TFs with different expression abundances were detected in the soapberry pericarp, which were classified into 54 families according to the Plant Transcription Factor Database 
(PlantTFDB) (Figure 3A). The MYB, NAC, WRKY, Nin-like, and ERF families of TFs accounted for the largest numbers of expressed TFs, with 201, 181, 180, 164, and 110 members, respectively. Dynamic analysis showed that most TF families had high expression levels at stages S1-S4. Meanwhile, some TF families had higher FPKM levels than other TF families, such as ARF, bZIP, CAMTA, DBB, Dof, MYB, and WRKY (Figure 3A). In addition, the expression patterns of TFs from clusters a, $d$, and f by Mfuzz clustering were consistent with the accumulation of DASs (clusters A, B, C), including 130 MYBs, 114 WRKYs, 67 ERFs, 57 bHLHs, and 36 bZIPs, suggesting that these TFs may play important roles in regulating saponin biosynthesis in soapberry (Figure 3B).

\section{Co-expression network analyses identified saponin-related genes}

To obtain further insight into the triterpenoid saponin biosynthesis in soapberry, WGCNA was performed to investigate the co-expression networks of all expressed genes. After deletion and outlier values were eliminated, 18,717 genes remained. These genes were clustered into 24 major tree branches, each of which represented a module (labeled with different colors; the grey module contains the remaining uncorrelated genes) (Figure 4A; Supplemental Table S6). Analysis of module-saponin relationships showed that the blue and greenyellow modules were significantly correlated with most saponins, with high correlation coefficients (Figure 4B). The blue module with 5211 genes was highly positively correlated with saponins 1, 2, and saponin 27, and highly negatively correlated with total saponins and 15 other saponins (including saponins 19, 20, and 22). The greenyellow module contained 3090 genes and showed a significant association with saponins 9, 12, 30, 32, and 54. Therefore, we selected these two modules (containing 8301 genes) positively or negatively correlated with saponin biosynthesis for further study. Genes preferentially expressed in the early stages of fruit growth (stages S1-S4) were mainly accumulated in the blue module (Figure 4C), while most of the genes highly expressed in the fully developed and mature stage (stage S8) were located in the greenyellow module (Figure 4D). Although both of these modules showed strong correlations with saponins, the genes in the two modules had different expression patterns, suggesting that the genes in these two modules may be involved in different functions in the biosynthesis of saponins. In addition, the patterns of 
clustering analysis, where 2442 genes (cluster 2) were found to be highly expressed in the early stages of fruit growth (stage S1) and 3816 genes (cluster 11) were highly expressed in stage S8 (Figure 4D). In addition, most of the genes in these two modules were also identified in the selected Mfuzz clusters. Thus, both WGCNA and Mfuzz clustering have identified two clusters/modules consistent with saponin accumulation patterns despite the different algorithms.

Further, the results of KEGG analysis results showed that the genes in the blue module were primarily related to glycosyltransferases, photosynthesis, photosynthesis proteins, and cytoskeleton proteins (Figure 4C), which were strongly correlated with saponins, suggesting that glycosyltransferases and photosynthetic metabolites may play important roles in saponin biosynthesis. This is similar to the conclusion that the photosynthetic metabolites (glucose and fructose) contribute to the synthesis of ginsenosides or movement into ginseng roots reported by schramek et al (Schramek et al., 2014). In addition, 17 saponin biosynthetic structural genes, including HMGR, DXS, GPS and $\beta$-AS, were enriched in terpenoid backbone biosynthesis and sesquiterpenoid and triterpenoid biosynthesis. Meanwhile, another 27 saponin biosynthesis candidate genes, including CYP450s and UGTs, were also found in the blue module. These results further verified that the co-expressed genes in this module are involved the accumulation of saponins in the soapberry pericarp. In the greenyellow module, the genes were significantly enriched for ribosome biogenesis, messenger RNA biogenesis, transcription machinery, and basal transcription factors (Figure 4D), suggesting that the genes in this module may regulate the biosynthesis of saponins.

\section{Candidate hub genes related to saponin biosynthesis}

Module hub genes are usually considered as representatives of given modules in biological networks. In the blue module, 46 genes showed strong correlations (edge weight $\geq 0.35$ ) (Supplemental Table S7; Supplemental Figure S9A). Among them, kinesin family member 4/21/27 protein (KIF4_21_27, Samuk02G0147800), putative protein kinase RLK-Pelle-LRR-XIIIb family protein (RPLX1, Samuk12G0107600), INO80 complex subunit B protein (INO80B, Samuk13G0014100), Samuk05G0034700 (unknown protein), cyclin D3 (CYCD3, Samuk02G0146500), LRR receptor-like serine/threonine-protein kinase ERECTA (ER, Samuk06G0040200), putative X8 domain-containing protein (X8DC1, Samuk05G0140500), and 
SmCYP71D-3 (NewGene.20862) had the highest degree of connectivity in the network, suggesting that these genes may play important roles in saponin biosynthesis. The network diagram of the greenyellow module is composed of 42 co-expressed genes (edge weight $\geq 0.45$ ), in which genes such as the BES1 family protein (SmBES1-7, Samuk03G0151400), protease

Do-like 9 (PD9, Samuk10G0098700), DNA-directed RNA polymerase (RPO41, Samuk09G0117400), transcription elongation regulator 1 (CA150, Samuk07G0143300), and MYB family protein (SmMYB57, Samuk08G0065200) have the highest degree of connectivity (Supplemental Table S8; Supplemental Figure S9B), suggesting that these genes may also participate in saponin biosynthesis. However, this requires further verification.

\section{Generation of the saponin biosynthesis regulatory network}

To construct the regulatory network associated with saponin biosynthesis, we screened the structural genes involved in the saponin biosynthesis pathway identified in the blue and greenyellow modules. We identified 44 structural genes associated with saponin biosynthesis in the blue module, the expression levels of which were strongly correlated with the accumulation of saponins (Supplemental Table S9). By associating the expression patterns and the potential binding affinity for the promoters of these saponin biosynthesis-related genes, we identified 40 TFs, including ERF, TCP, SBP, Trihelix, CPP, bHLH, and WRKY - the expression levels of which were strongly correlated with the 30 saponin biosynthesis-associated genes in the blue 273 module - and generated a correlated network (Supplemental Table S10; Figure 5). These 274 observations indicated that these TFs correspond to the putative regulators controlling saponin 275 biosynthesis in soapberry pericarp. As only four saponin biosynthesis candidate genes were identified in the greenyellow module (Supplemental Table S11), no eligible structural TF-gene pairs were detected. In addition, the results of qRT-PCR showed that the expression levels of multiple TFs in the blue module exhibited strong correlations (Pearson correlation coefficient, $\mathrm{r}>$ 0.6) with structural genes-for example, SmHMGR4, SmCYP71D-3, SmCYP716A-5, and SmUGT91A-2 - thus further verifying the reliability of the regulatory network(Figure 6). 
As structural modification of the triterpenoid backbone is one of the most important steps in the biosynthesis of triterpenoid saponins, we focused our analysis on the regulatory network of CYP450 genes responsible for oxidizing the backbones created by OSCs into sapogenins. There were nine CYP450s associated with saponin biosynthesis in the TF-structural gene regulatory network in the blue module (Figure 5). To identify the key TFs modulating activation of the candidate genes encoding CYP450s, we focused on the regulation of $S m C Y P 71 D-3$ because this candidate gene exhibited high levels of transcript accumulation as well as expression specificity and was identified as a DEG and a hub gene in the blue module (Supplemental Figure S10). This regulatory network analysis suggested that $S m C Y P 71 D-3$ is highly correlated with $29 \mathrm{TFs}$, including the ERF, TCP, SBP, bHLH, CPP, Trihelix, and WRKY families (Supplemental Table S12; Supplemental Figure S10). We randomly selected nine of these TFs as candidates for further study, namely, SmbHLH2, SmCPP1, SmERF25, SmERF29, SmSBP1, SmSBP4, SmTCP4, SmTrihelix6, and SmWRKY27, which showed the strongly correlated expression, high expression levels, and the ability to bind to the promoter of $S m C Y P 71 D$-3 (Figure 7A; Supplemental Figure S10). Further, we conducted yeast one-hybrid (Y1H) assays to investigate direct binding of these nine TFs to the $S m C Y P 71 D-3$ promoter. The fragment containing the core motifs of the SmCYP71D-3 promoter was cloned into the upstream of the Aureobasidin (AbA) resistance gene and transformed into the Y1H Gold strain as bait. In addition, the basal activity of the Y1H Gold strain transformed with pAbAi-SmCYP71D-3 was successfully inhibited in the presence of $50 \mathrm{ng} / \mathrm{mL}$ AbA (Figure 7A). We expressed these TFs fused to the GAL4 AD in the Y1H system to challenge them with the SmCYP71D-3 promoter fused to the $A b A r$ reporter. Among the nine TFs, only the yeast transformed with SmbHLH2, SmTCP4, and SmWRKY27 grew well in the selection medium, suggesting that these three TFs could induce SmCYP71D-3 promoter activity (Figure 7A). Consistent with these observations, electrophoretic mobility shift assays (EMSAs) suggested that SmbHLH2, SmTCP4, and SmWRKY27 bound to the DNA probes containing the corresponding TF binding sites present in the promoter region of SmCYP71D-3 (Figure 7B). In conclusion, these results support the hypothesis that SmbHLH2, 
regulation of the transcription of $S m C Y P 71 D-3$ in soapberry pericarp.

\section{Discussion}

312 Soapberry pericarp has been used as medicine and as an additive for cosmetics due to its

313 abundant triterpenoid saponins with several bioactivities and abilities as foaming agents.

314 However, very little is known about saponin accumulation or the molecular mechanism of saponin biosynthesis in soapberry pericarp. In this study, the differences in saponins at different growth stages of soapberry pericarp were analyzed qualitatively and quantitatively analyzed by UHPLC-Q-Qrbitrap-MS (saponin targeted). Metabolite profiling tentatively identified 54 saponins, significantly increasing our understanding of the saponins in soapberry pericarp. Saponin-producing plants usually accumulate saponins as part of their normal development. Meanwhile, saponin accumulation is also affected by many environmental factors, including nutrient and water availability, light irradiation, diseases, and pests (Augustin et al., 2011). Ndamba et al. (1994) suggested that maximal saponin accumulation in the early stages of Phytolacca dodecandra berry growth is to prevent fruit loss and ensure seed maturation. We obtained similar results indicating that the most saponins in soapberry pericarp accumulated rapidly in stage S2 and reached the highest level in stage S4 (Figure 1), accompanying by two fruit loss stages (Zhao et al., 2019). Meanwhile, the precipitation and temperature in the orchard were highest at stage S4 (Zhao et al., 2019). It was inferred that variation in saponin levels in the soapberry pericarp may be related to protection and responses to environmental factors.

Plant triterpenoid saponins have a wide variety of complex structures (Augustin et al., 2011). Although they have a common precursor synthesis pathway, the post-modification process from 2,3-oxidosqualene to the formation of the final triterpenoid saponin has various rules in different plants (Haralampidis et al., 2002; Augustin et al., 2011). This process mainly involves three enzymes, namely, OSCs, CYP450s, and UGTs. Cyclization of 2,3-oxidized squalene is considered the first committed step in the triterpenoid saponin biosynthesis. Then, the basal sapogenin backbone formed by OSCs usually undergoes hydroxyl, ketone, aldehyde and carboxyl modification by CYP450s before glycosylation. Finally, the saccharide side chains are 
of 135 saponin biosynthesis candidate genes were annotated in the persent study, including 7 OSCs, 41 CYP450s, and 45 UGTs. Among them, four $\beta$-ASs (SmbAS1, SmbAS3, SmbAS4, and SmbAS6) showed identity to Betula platyphylla var. japonica BPY (Zhang et al., 2003), Lotus japonicus LjAMY1 (Sawai et al., 2006), and Glycyrrhiza glabra GgbAS1 (Hayashi et al., 2001), suggesting that they may encode $\beta$-amyrin synthase catalyzing conversion of 2,3 -oxidosqualene to $\beta$-amyrin (Supplemental Figure S6). Another candidate $O S C, S m L U S$, showed a high degree of identity to B. platyphylla var. japonica BPW (Zhang et al., 2003), L. japonicus OSC3 (Sawai et al., 2006), and G. glabra GgLUS1 (Hayashi et al., 2004), suggesting that this OSC may be responsible for the biosynthesis of lupane-type triterpenoid saponin in soapberry pericarp. Furthermore, the CYP450 enzymes, which catalyze the oxidation of $\beta$-amyrin or lupeol, especially at C-3, C-23, and C-28, are required for the biosynthesis of the main triterpenoid saponins in soapberry pericarp (Xu et al., 2018). CYP716As have been shown to be mainly responsible for catalyzing sequential three-step oxidation of the $\alpha$-amyrin/ $\beta$-amyrin/lupeol backbone to form hydroxyl, aldehyde and carboxyl moieties at the C-28 position (Ghosh, 2017; Miettinen et al., 2017; Xu et al., 2021). In this study, two CYP716As, SmCYP716A-2 and SmCYP716A-3, showed some similarity to Chenopodium quinoa CqCYP716A78 (Fiallos-Jurado et al., 2016) and Aquilegia coerulea AcCYP716A110 (Fiallos-Jurado et al., 2016) which catalyze $\beta$-amyrin to oleanolic acid, suggesting that they have the same catalytic activities in soapberry pericarp (Supplemental Figure S7). We also found that three candidate CYP72As (SmCYP72A-1, SmCYP72A-5, and SmCYP72A-7) showed identity to Kalopanax septemlobus KsCYP72A397, (Han et al., 2017) which catalyzes the hydroxylation of oleanolic acid at C-23, suggesting that these CYP72As may catalyze oleanolic acid to hederagenin in soapberry (Supplemental Figure S7). In addition, four CYP71Ds, SmCYP71D-1, SmCYP71D-2, SmCYP71D-3, and SmCYP71D-4, showed identity to $L j C Y P 71 D 353$, which catalyzes the C-20 hydroxylation of dihydro-lupeol and C-28 oxidation of 20-hydroxy-lupeol in L. japonicus (Krokida et al., 2013), suggesting that these candidate CYP71Ds may participate in the biosynthesis of lupane-type triterpenoid saponins in soapberry (Supplemental Figure S7). UGTs participating in saponin biosynthesis belonging to the UGT 71, 73, 74, 85, and 91 clans were identified previously (Rahimi et al., 2019). The UGT73 clan is the best candidate group for oleanane-type triterpenoid saponin biosynthesis (Erthmann et al., 2018). We identified three genes (SmUGT73C-9, SmUGT73C-10, and 
SmUGT73C-11) belonging to the UGT73 clan that showed high degrees of identity to Panax japonicus var. bipinnatifidus PjOAGT and Panax zingiberensis PzOAGT1, PzOAGT2, and PzOAGT3 (Supplemental Figure S8), which transfer glucuronic acid at C-3 of oleanolic acid to form oleanolic acid 3-O- $\beta$-glucuronide (Tang et al., 2019), suggesting that these genes may catalyze the glucuronosylation of the C3-hydroxyl group for the biosynthesis of triterpenoid saponins in soapberry. In addition, four candidate UGT74s (SmUGT74G-1, SmUGT74G-2, SmUGT74G-3, and SmUGT74G-4), two candidate UGT94s (SmUGT94E-1 and SmUGT94E-2), and six candidate UGT71s (SmUGT71D-1, SmUGT71D-2, SmUGT71D-3, SmUGT71D-4, SmUGT71K-1, and SmUGT71K-4) may be responsible for the glycosylation of tetracyclic sapogenins in soapberry (Dai et al., 2015; Wang et al., 2015; Wei et al., 2015; Lu et al., 2017; Lu et al., 2017). Further studies are needed to determine the functions of these candidate genes in the triterpenoid saponin biosynthesis pathway, including the key intermediates, in soapberry.

Combining these transcriptomic and metabolomic resources allowed the identification of key structural and regulatory genes involved in triterpenoid saponin biosynthesis in soapberry. This study not only validated some previously reported metabolic regulatory network information, but also identified novel TFs that regulate triterpenoid saponin biosynthesis. Previous studies have identified two bHLH family TFs, TSAR1 and TSAR2, from Medicago truncatula, that could instigate the expression of HMGR1, $\beta$-AS, CYP93E2, CYP72A61v2, UGT73K1, and UGT73F3 as well as MAKIBISHI1, $\beta$-AS, CYP716A12, CYP72A68v2, and UGT73F3, resulting in corresponding accumulation of nonhemolytic and hemolytic soyasaponins, respectively (Mertens et al., 2016). In this study, we verified the relatively high correlation of expression ( $\mathrm{r}=0.96)$ between $S m b H L H 2$ and $S m C Y P 71 D-3$, which suggested the reliability of our analysis to identify regulators that control saponin biosynthesis. Y1H assay and EMSA also confirmed the transcriptional regulatory action of SmbHLH2 on SmCYP71D-3. In addition, the correlations in expression between SmCPP1, SmERF25, SmERF29, SmSBP1, SmSBP4, SmTCP4, SmTrihelix6, SmWRKY27, and SmCYP71D-3 were also high $(r>0.80)$. Considering the much higher expression levels of these TFs throughout pericarp development, we proposed that these TFs may be key regulators involved in modulating triterpenoid saponin accumulation. qRT-PCR results also showed that the expression pattern of SmCYP71D-3 was similar to those of SmCPP1, SmERF25, SmERF29, SmSBP1, SmSBP4, SmTCP4, SmTrihelix6, 
and SmWRKY27 (Figure 6). However, Y1H assay and EMSA results showed that only SmTCP4 and SmWRKY27 would be able to bind to $S m C Y P 71 D-3$ via the predicted cis-motif elements, indicating that the results of bioinformatics prediction included certain false positives. The physiological function of SmCYP71D-3 and corresponding TFs in saponin metabolism and the transcriptional regulation of these TFs on $S m C Y P 71 D-3$ by these TFs require further study. Further investigations focusing on the functions of other structural and regulatory genes would help to elucidate the molecular regulatory mechanisms of soapberry pericarp saponin biosynthesis.

\section{Materials and methods}

\section{Plant materials}

For this experiment, three 10-year-old soapberry superior trees were selected for sampling. The details of these plant materials were described in our previous report (Xu et al., 2021). The plant materials were cultivated at soapberry national forest germplasm banks in Jianning County, Fujian Province, China under natural conditions. Fruit samples were collected at eight growth stages between June and November 2018: early ovary development (stage S1), approximately 15 days after pollination (DAP); 30\% of the largest fruit size (stage S2), 45 DAP; 70\% of the largest fruit size (stage S3), 75 DAP; 80\% of the largest fruit size (stage S4), 90 DAP; 90\% of the largest fruit size (stage S5), 105 DAP; beginning of maturity (stage S6), 120 DAP; great change in pericarp (stage S7), 135 DAP; fully developed and mature (stage S8), 150 DAP. After picking

417 the fruit, we immediately separated the pericarp from the seed. A portion of each pericarp sample 418 was transferred to liquid nitrogen, and finally stored at $-80{ }^{\circ} \mathrm{C}$ for RNA extraction. The other 419 portion of each sample was dried to a constant weight in an oven at $40^{\circ} \mathrm{C}$ and then ground into 420 uniform powder with a ball mill (MM400; Retsch, Arzberg, Germany) for saponin 421 determination.

\section{Total saponin measurement and saponin metabolite profiling}

The total saponin content was measured by the vanillin-glacial acetic acid method, as 
described by Sun et al. (2017). Absorbance was measured with a UV-Vis spectrometer (Lambda 35; PerkinElmer, Wellesley, MA, USA) at $547 \mathrm{~nm}$. All experiments were performed in three biological replicates and three technical replicates.

The relative quantities of saponin metabolites in soapberry pericarp samples were analyzed by ultra-high-performance liquid chromatography with a Q Exactive Hybrid Quadrupole Orbitrap High-Resolution Accurate Mass Spectrometry (UHPLC-Q-Qrbitrap-MS) system by Shanghai Profleader Biotech Co., Ltd., Shanghai, China) (refer to Supplemental Method S1 for details regarding the protocol).

\section{RNA extraction, library construction, and sequencing}

Total RNA extraction was performed using a FinePure Plant RNA Kit (Genfine Biotech Co. Ltd., Beijing, China) and the qualities were assessed using a Bioanalyzer 2100 (Agilent, Palo Alto, CA, USA). A cDNA library was constructed using a NEBNext ${ }^{\circledR}$ Ultra $^{\mathrm{TM}}$ RNA Library Prep Kit for Illumina ${ }^{\circledR}$ (New England Biolabs, Ipswich, MA, USA) in accordance with the manufacturer's protocol. A Qubit fluorometer ${ }^{\circledR}$ (Thermo Fisher Scientific, Waltham, MA, USA) and Qseq100 DNA Analyzer (Bioptic Inc., San Francisco, CA, USA) were used to determine the quality of the library based on the criteria of DNA concentration was $>1.0 \mathrm{ng} / \mu \mathrm{L}$ and the DNA length $\sim 400 \mathrm{bp}$. The DNA molar concentration of the library was quantified with the KAPA Library Quantification Kit Illumina ${ }^{\circledR}$ Platform (KAPA Biosystems, Woburn, MA, USA). Libraries were sequenced using an Illumina HiSeq ${ }^{\circledR} \mathrm{X}$ Ten System with paired-end reads by Beijing Yuanyi Biotechnology Co., Ltd. (Beijing, China).

\section{Analysis of RNA-Seq data}

First, the adapter sequences, reads containing Ns (uncertain base information), and low-quality sequence reads were removed from the raw reads to produce clean reads. These clean reads were then aligned to the soapberry reference genome (Unpublished) sequence to acquire the genes using HISAT2 (Kim et al., 2015) and StringTie software (Pertea et al., 2015). The transcripts consistent with the reference genome were called known genes, and other new transcripts were called novel genes (prefixed with "NewGene"). The annotation information of genes was 
obtained from the soapberry genome annotation project. In addition, we also aligned the genes to annotated protein sequences of two species containing triterpenoid saponin, Medicago truncatula (http://www.medicagogenome.org/) and Glycine max (https://www.soybase.org/sbt/) (BLASTX; e-value $\leq 1 \mathrm{e}-5)$. To analyze candidate TFs in soapberry, nucleotide sequences were compared with the sequences in the Plant Transcription Factor Database (PlantTFDB) (http://planttfdb.gao-lab.org/index.php) using the default parameters (Tian et al., 2019).

Gene expression levels were calculated and normalized to fragments per kilobase of transcript per million fragments mapped (FPKM) (Trapnell et al., 2010). The differentially expressed genes (DEGs) between the samples were identified with DESeq2 (1.20.0) (Anders and Huber, 2010; Love et al., 2014). Genes with an Benjamini-Hochberg-adjusted $p$-values ( $Q$-values) $<0.05$ and $\mid \log _{2}$ (fold change) $\mid>1$ were considered to be differentially expressed genes (DEGs). The identified DEGs were further subjected to enrichment analysis through Gene Ontology (GO) annotation and Kyoto Encyclopedia of Genes and Genomes (KEGG) pathway analysis using the clusterProfiler R package.

The deduced amino acid sequences of the $O S C, C Y P 450$, and $U G T$ genes of soapberry in this study and those of other plants obtained from GenBank were used for phylogenetic analysis. Multiple sequence alignments were generated using Clustal W program. Phylogenetic analysis was carried out using the Neighbor-joining method with MEGA-X software program. A bootstrap of 1000 replications was used to estimate the strength of nodes in the tree.

\section{WGCNA and gene network visualization}

The gene co-expression networks were constructed by the WGCNA method implemented in R software based on all expressed genes in our study (Langfelder and Horvath, 2008). The co-expression modules were constructed using the automatic network construction function 474 (blockwiseModules) with default parameters, except for the soft thresholding power of 9, mergeCutHeight of 0.25 , deepSplit of 2 , and minModuleSize of 30 . FPKM values were normalized, and an adjacency matrix was constructed. The resulting adjacency matrix was converted to a topological overlap matrix (TOM). After constructing a network, the genes with

478 similar expression patterns were grouped into the same modules, and eigengenes were also calculated for these modules. Eigengene values were used to search the associations with 
saponins produced during fruit development. Then, cis-motif enrichment analysis of the TFs and saponin biosynthesis candidate genes in the chosen modules was conducted as described by Wang et al. (2021). First, the position frequency matrices (PFMs) of TFs were downloaded from plantTFDB (Tian et al., 2019); FIMO was then used to predict the cis-motif information in the promoter region (2000 bp upstream and $200 \mathrm{bp}$ downstream of the transcriptional start site) of the saponin biosynthesis candidate genes under the condition $p<1 \mathrm{e}-3$ (Grant et al., 2011). Finally, we constructed transcriptional regulatory networks by combining the availability of cis-acting element binding sites present in the promoters of the saponin biosynthesis candidate genes and the Pearson correlation coefficient $(r>0.8)$ between these candidate genes and TFs in the same WGCNA module. In addition, the networks were visualized using the Cytoscape (v3.8.2) (Shannon et al., 2003).

\section{qRT-PCR analysis}

Total RNA from pericarp at eight stages of growth was prepared according to the protocol described above. The first-strand cDNA was synthesized using a GoldenstarTM RT6 cDNA Synthesis Kit Ver 2 (Tsingke, Beijing, China) with reverse transcriptase. Eleven candidate genes and five TFs involved in saponin biosynthesis identified in the RNA-Seq data were selected for qRT-PCR analysis, and SmACT (Samuk13G0061200) was used as a reference to normalize gene expression. All primers used in qRT-PCR are listed in Supplemental Table S13. Reactions were performed on a LineGene 9600 Plus quantitative Real-Time PCR detection system (Bioer, Hangzhou, China) using a T5 Fast qPCR Mix (SYBR Green I) (Tsingke). Three biological replicates and three technical replicates were carried out for each stage. The relative expression level of each gene was determined by calculating the fold change in selected stages of pericarp samples relative to stage $\mathrm{S} 1$ using the $2^{-\Delta \Delta \mathrm{Ct}}$ method.

\section{Yeast one-hybrid (Y1H) assays}

Y1H assays were carried out based on the method described previously by Mou et al. (2018). Fragments of the target gene $(S m C Y P 71 D-3)$ promoter containing the core cis-elements were directly synthesized (Genecreate, Wuhan, China) and cloned into the pAbAi vector. The resulting 
pAbAi-SmCYP71D-3 plasmids produced were then linearized and transformed into the $\mathrm{Y} 1 \mathrm{H}$

Gold strain and selected with medium lacking Leu and supplemented with AbA (SD/-Leu/AbA medium). The open reading frames of TFs were inserted into the pGADT7 vector and transformed into Y1H Gold strains containing pAbAi-SmCYP71D-3. The empty pGADT7 vector and pGADT7-P53 were used as negative and positive controls, respectively. The transformants were further cultivated in medium lacking Leu (SD/-Leu medium). Positive yeast clones were grown on SD/-Leu liquid medium and diluted to different concentrations $\left(\mathrm{OD}_{600}=10^{-1}, 10^{-2}\right.$,

$51410^{-3}, 10^{-4}$ ), and aliquots of $10 \mu \mathrm{L}$ yeast suspensions were plated on the SD/-Leu/AbA plates. All transformation and screening procedures were conducted twice.

\section{Electrophoretic mobility shift assay (EMSA)}

517

518

519

520

521

522

523

524

525

526

527

528

529

The full-length coding sequences of SmbHLH2, SmTCP4, and SmWRKY27 were amplified and inserted into the pGEX4T-1 vector to generate GST fusion proteins. SmbHLH2-GST, SmTCP4-GST, and SmWRKY27-GST were purified using a NucBusterTM Protein Extraction Kit (Cat. \#71183; Merck KGaA, Darmstadt, Germany) for use in further EMSA experiments. The 26-nt probes of the promoter containing the putative cis-element binding sites of SmCYP71D-3 were synthesized and labeled with biotin at the 5' end (Genecreate). Mutant probes and unlabeled cold probes (20-, 50-, 100-fold concentration of unlabeled oligonucleotides) were utilized for competition experiments. The probes were incubated with the nuclear extract at room temperature for $30 \mathrm{~min}$. The entire reaction mixture was run on a nondenaturing $0.5 \times \mathrm{TBE} 6 \%$ polyacrylamide gel for $1 \mathrm{~h}$ at $60 \mathrm{~V}$ and $4^{\circ} \mathrm{C}$, and then transferred onto Biodyne $® \mathrm{~B}$ nylon membranes (Pall Corp., Ann Arbor, MI, USA) (Hellman and Fried, 2007). Signals were visualized with reagents included in the Efficient Chemiluminescence Kit (Vigorous, Beijing, China) and ChemiDoc XRS (Bio-Rad, Hercules, CA, USA).

\section{Statistical analyses}

One-way analysis of variance (ANOVA) and Duncan's multiple comparison test were used to analyze the differences in total saponin content at different stages using SPSS software (version 25.0; IBM Inc., Chicago, IL, USA). In all analyses, $p<0.05$ was taken to indicate statistical 
significance. Charts were plotted using Origin 2017 SR2 software (OriginLab Inc., Hampton, MA, USA). Correlation analysis, hierarchical clustering and principal component analysis (PCA) were performed using the corrplot and prcomp package in $\mathrm{R}$.

\section{Accession numbers}

Sequence data have been deposited in National Center for Biotechnology Information (NCBI)

Sequence Read Archive (http://www.ncbi.nlm.nih.gov/sra) with the BioProject ID

\section{Supplemental data}

542

Supplemental Figure S1. qRT-PCR verified the selected genes. Relative expression levels of qRT-PCR were calculated using SmACT (Samuk13G0061200) as a standard. Normalized gene levels in stage S1 were arbitrarily set to 1 . Pearson correlation coefficients were calculated by comparing qRT-PCR and RNA-seq data for each gene across all samples.

Supplemental Figure S2. Number of DEGs between two tested groups $(Q$-value $<0.05$ and $\mid \log _{2}$ (Fold Change $\left.) \mid>1\right)$. Here, FC refers to the $\log 2$ of the ratio of gene expression between two tested groups.

Supplemental Figure S3. GO enrichment analysis of DEGs between eight pericarp growth stages. Top 50 GO categories assigned to the DEGs. The genes were categorized based on gene ontology annotation and the proportion of each category is display in the categories of biological process $(\mathrm{BP})$, cellular component $(\mathrm{CC})$ and molecular function $(\mathrm{MF})$.

Supplemental Figure S4. Temporal expression patterns of DEGs in the eight growth stages of the soapberry pericarps. All DEGs were classified into twelve clusters based on their expression patterns using the Mfuzz R package.

Supplemental Figure S5. Distribution of enriched KEGG pathways for various DEGs expression patterns in the pericarp growth. KEGG pathways involved in the biosynthesis of metabolites of cluster 2 (A), cluster 9 (B) and cluster 11 (C). Each circle represents a KEGG 
pathway.

560 Supplemental Figure S6. Phylogenetic tree of the soapberry $\beta$-ASs and LUS. The phylogenetic tree is constructed based on the deduced amino acid sequences for the soapberry $\beta$-ASs and LUS (bold letters) and other plant $\beta$-ASs and LUS involved in triterpenoid biosynthesis via the Neighbor-Joining method and established the reliability of each node through bootstrap methods, using ClustalW. Protein sequences are retrieved from NCBI GenBank using the following accession numbers: Lotus japonicus LjAMY1 (AB181244.1), LjAMY2 (AF478455.1) and OSC3 (AB181245.1); Glycyrrhiza glabra GgbAS1 (AB037203.1) and GgLUS1 (AB116228.1); Betula platyphylla var. japonica BPY (AB055512.1) and BPW (AB055511.1); Panax ginseng PNY1 (AB009030.1) and PNY2 (AB014057.1); Eleutherococcus senticosus EsBAS (KX378998.1); Barbarea vulgaris LUP2 (KP784688.1); Arabidopsis thaliana AtBAS (AB374428.1); Vaccaria hispanica VhBS (DQ915167.1); Chenopodium quinoa CqbAS1 (KX343074.1).

Supplemental Figure S7. Phylogenetic tree of the soapberry CYP450s. The phylogenetic tree is constructed based on the deduced amino acid sequences for the soapberry CYP450s (bold letters) and other plant CYP450s involved in triterpenoid biosynthesis via the Neighbor-Joining method and established the reliability of each node through bootstrap methods, using ClustalW. Protein sequences are retrieved from NCBI GenBank using the following accession numbers: $L$. japonicus LjCYP71D353 (KF460438.1); Platycodon grandiflorus $\operatorname{PgCYP716A141~}$

577

578 (KU8878855.1); Artemisia annua AaCYP716A14v2 (KF309251.1); C. quinoa CqCYP716A78 (KX343075.1); Aquilegia coerulea AcCYP716A110 (KU878864.1); Kalopanax septemlobus KsCYP72A397 (KT150517.1); Medicago truncatula MtCYP72A63 (AB558146.1) and MtCYP72A68v2 (DQ335782.1); Glycine max GmCYP72A69 (LC143440.1); Glycyrrhiza uralensis GuCYP72A154 (AB558153.1).

Supplemental Figure S8. Phylogenetic tree of the soapberry UGTs. The phylogenetic tree is constructed based on the deduced amino acid sequences for the soapberry UGTs (bold letters) and other plant UGTs involved in triterpenoid biosynthesis via the Neighbor-Joining method and established the reliability of each node through bootstrap methods, using ClustalW. Protein sequences are retrieved from NCBI GenBank using the following accession numbers: $V$. hispanica VhUGT74M1 (DQ915168.1); Panax japonicus var. bipinnatifidus PjOAGT 
(MH819287.1); Panax zingiberensis PzOAGT1 (MH819284.1), PzOAGT2 (MH819285.1) and PzOAGT3 (MH819286.1); Panax quinquefolius Pq3-O-UGT1 (KR028477.1) and Pq3-O-UGT2

590

591

592

593

594

595

596

597

598

599

600

601

602

603

604

605

606

607

608

609

610

611

612

613

614

615

616 Supplemental Method S1. Supplemental materials and methods.

(KR106207.1); Siraitia grosvenorii SgUGT74AC1 (HQ259620.1), SgUGT720-269-1 and SgUGT94-289-3; P. ginseng PgUGTPg45 (KM401918.1), PgUGTPg100 (KP795113.1) and PgUGTPg101 (KP795114.1).

Supplemental Figure S9. The correlation networks of genes in the blue (A) and greenyellow (B) modules, in which only edges with weight above a threshold of 0.35 and 0.45 are displayed, respectively.

Supplemental Figure S10. Transcriptional regulatory network of SmCYP71D-3.

Supplemental Table S1. Triterpenoid saponin metabolites tentatively detected in soapberry pericarps of different fruit growth stages.

Supplemental Table S2. Differentially accumulated saponins between different fruit growth stages.

Supplemental Table S3. The results of Mfuzz clustering for all of DASs in the eight fruit growth stages of the soapberry pericarps.

Supplemental Table S4. Summary of RNA sequencing and mapping using the soapberry genome as a reference.

Supplemental Table S5. List of saponin biosynthesis candidate genes.

Supplemental Table S6. Module sizes of WGCNA.

Supplemental Table S7. List of genes in blue module correlation network (with edge weight $\geq$ $0.35)$.

Supplemental Table S8. List of genes in greenyellow module correlation network (with edge weight $\geq 0.45$ ).

Supplemental Table S9. Gene annotation in formations of blue module.

612 Supplemental Table S10. Network nodes of structural TF-gene network in the blue module.

13 Supplemental Table S11. Gene annotation in formations of greenyellow module.

14 Supplemental Table S12. Network edges of structural TF-gene network in the blue module.

15 Supplemental Table S13. Primers of sequences for qRT-PCR analysis. 
618 We sincerely thank Shuijing Luo for his tending of the plants. We are also grateful to Kunjing

$619 \mathrm{Wu}$ and Kui Liu for his guidance in the experiment. We gratefully acknowledge the assistance of 620 Xin Wang and Jing Zhong in this study.

\section{Funding}

622

623

624

625

626

627

628

629

630

631

632

633

634

635

636

637

638

639

This work was supported by the National Natural Science Foundation of China (No. 32071793) and the Special Foundation for National Science and Technology Basic Research Program of China (No. 2019FY100803).

\section{Conflict of interest statement}

The authors declared that they have no conflicts of interest to this work.

\section{Figure legends}

Figure 1. Images of soapberry fruits and dynamic changes of saponins in the pericarp at eight growth stages.

(A) Images of soapberry fruits and pericarps at eight growth stages. (B) Dynamic changes of total saponin contents of soapberry pericarp at eight growth stages. Means \pm SD ( $n=3)$. Means in different letters $(\mathrm{a}-\mathrm{b})$ are significantly different at $P<0.05$ by ANOVA on Duncan's multiple range test. (C) Principal component analysis (PCA) of detected saponins. (D) Hierarchical clustering heat map of detected saponins. (e) Upper: Temporal expression patterns of DASs. All DASs were classified into three clusters based on their contents using the Mfuzz R package. (E) Lower: Heatmap of DASs accumulation level in cluster A, cluster B and cluster C.

Figure 2. Proposed pathways for triterpenoid saponin biosynthesis in soapberry. Expression levels of genes encoding enzymes that catalyze each step of the triterpenoid saponin biosynthesis 
pathway are shown. The higher and lower expression level of genes are indicated in firebrick and navy, respectively.

642

643 Figure 3. Expression dynamics of TF families.

644 (A) The colour shows the total FPKM of all TFs of a particular TF family. The first number in 645 parentheses is the number of DEGs in a TF family, and the second number refers to the total number of genes in that TF family as annotated in PlantTFDB. (B) Expression profiles of all TFs by Mfuzz clustering analysis.

648

Figure 4. WGCNA of all expressed genes.

(A) Hierarchical clustering tree (cluster dendrogram) showing 24 modules of co-expressed genes by WGCNA. Each leaf of tree corresponds to one gene. The major tree branches constitute 24 modules, labeled with different colors. (B) Module-saponin relationship. Each row represents a module. Each column represents a specific saponin (detailed information reference Table S4). The value in each cell at the row-column intersection represents the correlation coefficient between the module and the saponin and is displayed according to the color scale on the right. The value in parentheses in each cell represents the $P$ value. (C) Upper: heat map of genes in the blue module; Lower: the KEGG enrichment analysis of the genes in the blue module showed only the top 20 pathways with the most significant enrichment. (D) Upper: heat map of genes in the greenyellow module; Lower: the KEGG enrichment analysis of the genes in the greenyellow module showed only the top 20 pathways with the most significant enrichment.

Figure 5. The regulatory network of triterpenoid saponin biosynthesis in soapberry. Hexagon with different colors indicate different families of structural genes associated with saponin biosynthesis in the blue module. Circles with different colors indicate different families of TFs characterize in the same module which transcripts are highly correlated with expression of structural genes.

Figure 6. Expression correlation of TFs and their predicted target genes. The bars and lines indicate the relative expression level of TFs and their corresponding target genes in the eight 
675

growth stages of soapberry pericarps. The Y-axis on the left and right represents the abundance of the TFs and their target genes, respectively. SmACT was used for normalizing the relative expression of these encoding genes. The expression level of the genes in the stage S1 were set as 1.0. Relative expression level was calculated using the $2^{-\Delta \Delta C t}$ method. Data indicate the mean values of three biological replications.

Figure 7. Identification of SmbHLH2, SmTCP4, and SmWRKY27 as key TFs regulating SmCYP71D-3 in soapberry.

(A) Upper: schematic illustration of the potential recognition elements of the nine TFs in the $2 \mathrm{~kb}$ promoter region of $S m C Y P 71 D-3$. (A) Middle: no basal activities of ProSmCYP71D-3 were observed in yeast grown on SD medium lacking Leu in the presence of 50, 100, 200, 500, and $1000 \mathrm{ng} / \mathrm{mL}^{-1} \mathrm{AbA}$ (SD/-Leu/AbA). (A) Lower: yeast growth assays after Y1H reporter strains were transformed with effector containing the ORF of different TFs or empty (negative control). Interaction was determined based on the ability of transformed yeast to grow on SD medium lacking Leu in the presence of $50 \mathrm{ng} / \mathrm{mL}^{-1}$ (SD/-Leu/AbA). (B) SmbHLH2, SmTCP4, and SmWRKY27 binding to the promoter of SmCYP71D-3. The WT probe containing the corresponding TF binding site was biotin-labeled. Competition for TF binding was performed with cold probes. The symbols - and + represent absence or presence of the probes and GST-tagged TF protein. The core binding sequences are highlighted red; The upper sequence is the prediction TF binding site sequence, and the lower sequence is the mutation probe sequence.

\section{References}

Anders S, Huber W (2010) Differential expression analysis for sequence count data. Genome Biology 11: R106

Augustin JM, Kuzina V, Andersen SB, Bak S (2011) Molecular activities, biosynthesis and evolution of triterpenoid saponins. Phytochemistry 72: 435-457

Dai L, Liu C, Zhu Y, Zhang J, Men Y, Zeng Y, Sun Y (2015) Functional characterization of cucurbitadienol synthase and triterpene glycosyltransferase involved in biosynthesis of mogrosides from Siraitia grosvenorii. Plant Cell Physiol 56: 1172-1182

Deng B, Huang Z, Ge F, Liu D, Lu R, Chen CJJoPGR (2017) An AP2/ERF family transcription factor PnERF1 raised the biosynthesis of saponins in Panax notoginseng. $\mathbf{J}$ Plant Growth Regul: 691-701 
Erthmann PØ, Agerbirk N, Bak S (2018) A tandem array of UDP-glycosyltransferases from the UGT73C subfamily glycosylate sapogenins, forming a spectrum of monoand bisdesmosidic saponins. Plant Mol Biol 97: 37-55

Fiallos-Jurado J, Pollier J, Moses T, Arendt P, Barriga-Medina N, Morillo E, Arahana V, Torres M, Goossens A, Leon-Reyes A (2016) Saponin determination, expression analysis and functional characterization of saponin biosynthetic genes in Chenopodium quinoa leaves. Plant Sci 250: 188-197

Ghosh S (2017) Triterpene structural diversification by plant cytochrome P450 enzymes. Front Plant Sci 8: 1886

Grant CE, Bailey TL, Noble WS (2011) FIMO: Scanning for occurrences of a given motif. Bioinformatics 27: 1017-1018

Han JY, Chun JH, Oh SA, Park SB, Hwang HS, Lee H, Choi YE (2017) Transcriptomic analysis of Kalopanax septemlobus and characterization of KsBAS, CYP716A94, and CYP72A397 genes involved in hederagenin saponin biosynthesis. Plant Cell Physiol 59: 319-330

Haralampidis K, Trojanowska M, Osbourn AE (2002) Biosynthesis of triterpenoid saponins in plants. Adv Biochem Eng Biot 75: 31

Hayashi H, Huang P, Kirakosyan A, Inoue K, Hiraoka N, Ikeshiro Y, Kushiro T, Shibuya M, Ebizuka Y (2001) Cloning and characterization of a cDNA encoding $\beta$-amyrin synthase involved in glycyrrhizin and soyasaponin biosyntheses in licorice. Biol Pharm Bull 24: 912-916

Hayashi H, Huang P, Takada S, Obinata M, Inoue K, Shibuya M, Ebizuka Y (2004) Differential expression of three oxidosqualene cyclase mRNAs in Glycyrrhiza glabra. Biol Pharm Bull 27: 1086-1092

Hellman LM, Fried MG (2007) Electrophoretic mobility shift assay (EMSA) for detecting protein-nucleic acid interactions. Nat Protoc 2: 1849-1861

Jia L, Sun C (2012) Research progress of biodiesel tree Sapindus mukorossi. J. China Agric. Univ. 17: 191-196

Kim D, Langmead B, Salzberg SL (2015) HISAT: a fast spliced aligner with low memory requirements. Nature Methods 12: 357-360

Krokida A, Delis C, Geisler K, Garagounis C, Tsikou D, Peña-Rodríguez L, Katsarou D, Field B, Osbourn AE, Papadopoulou KK (2013) A metabolic gene cluster in Lotus japonicus discloses novel enzyme functions and products in triterpene biosynthesis. New Phytol 200: 675-690

Langfelder P, Horvath S (2008) WGCNA: an R package for weighted correlation network analysis. BMC Bioinformatics 9: 559-559

Li S (1975) Compendium of Materia Medica. People's Medical Publishing House, Beijing

Li Y, Chen Y, Zhou L, You S, Deng H, Chen Y, Alseekh S, Yuan Y, Fu R, Zhang Z, Su D, Fernie AR, Bouzayen M, Ma T, Liu M, Zhang Y (2020) MicroTom metabolic network: rewiring tomato metabolic regulatory network throughout the growth cycle. Mol Plant 13: $1203-1218$

Liu Y, Lv J, Liu Z, Wang J, Yang B, Chen W, Ou L, Dai X, Zhang Z, Zou X (2020) Integrative analysis of metabolome and transcriptome reveals the mechanism of color formation in pepper fruit (Capsicum annuum L.). Food Chem 306: 125629 
Love MI, Huber W, Anders S (2014) Moderated estimation of fold change and dispersion for RNA-seq data with DESeq2. Genome Biology 15: 550

Lu C, Zhao S, Wang X (2017) Functional regulation of a UDP-glucosyltransferase gene (Pq3-O-UGT1) by RNA interference and overexpression in Panax quinquefolius. Plant Cell Tiss Organ Cult 129: 445-456

Lu C, Zhao S, Wei G, Zhao H, Qu Q (2017) Functional regulation of ginsenoside biosynthesis by RNA interferences of a UDP-glycosyltransferase gene in Panax ginseng and Panax quinquefolius. Plant Physiol Bioch 111: 67-76

Meng J, Wang B, He G, Wang Y, Tang X, Wang S, Ma Y, Fu C, Chai G, Zhou G (2019) Metabolomics integrated with transcriptomics reveals redirection of the phenylpropanoids metabolic flux in Ginkgo biloba. J Agr Food Chem 67: 3284-3291

Mertens J, Pollier J, Bossche RV, Lopez-Vidriero I, Franco-Zorrilla JM, Goossens A (2016) The bHLH transcription factors TSAR1 and TSAR2 regulate triterpene saponin biosynthesis in Medicago truncatula. Plant Physiol 170: 194-210

Miettinen K, Pollier J, Buyst D, Arendt P, Csuk R, Sommerwerk S, Moses T, Mertens J, Sonawane PD, Pauwels L, Aharoni A, Martins J, Nelson DR, Goossens A (2017) The ancient CYP716 family is a major contributor to the diversification of eudicot triterpenoid biosynthesis. Nat Commun 8: 14153

Mou W, Li D, Luo Z, Li L, Mao L, Ying T (2018) SlAREB1 transcriptional activation of NOR is involved in abscisic acid-modulated ethylene biosynthesis during tomato fruit ripening. Plant Sci 276: 239-249

Ndamba J, Lemmich E, Mølgaard P (1994) Investigation of the diurnal, ontogenetic and seasonal variation in the molluscicidal saponin content of Phytolacca dodecandra aqueous berry extracts. Phytochemistry 35: 95-99

Nett RS, Lau W, Sattely ES (2020) Discovery and engineering of colchicine alkaloid biosynthesis. Nature 584: 148-153

Ni J, Zhao Y, Tao R, Yin L, Bai S (2020) Ethylene mediates the branching of the jasmonateinduced flavonoid biosynthesis pathway by suppressing anthocyanin biosynthesis in red Chinese pear fruits. Plant Biotechnol J 18: 1223-1240

Pertea M, Pertea G, Antonescu C, Chang TC, Mendell JT, Salzberg SL (2015) StringTie enables improved reconstruction of a transcriptome from RNA-seq reads. Nature Biotechnology 33: 290-295

Rahimi S, Kim J, Mijakovic I, Jung K, Choi G, Kim SC, Kim YJ (2019) Triterpenoid-biosynthetic udp-glycosyltransferases from plants. Biotechnol Adv 37: 107394

Sawai S, Shindo T, Sato S, Kaneko T, Tabata S, Ayabe SI, Aoki T (2006) Functional and structural analysis of genes encoding oxidosqualene cyclases of Lotus japonicus. Plant Sci 170: 247-257

Schramek N, Huber C, Schmidt S, Dvorski S, Kmispel N, Ostrozhenkova E (2014) Biosynthesis of ginsenosides in field-grown Panax ginseng. JSM Biotechnol Bioeng: 1033

Shannon P, Markiel A, Ozier O, Baliga NS, Wang JT, Ramage D, Amin N, Schwikowski B, Ideker T (2003) Cytoscape: a software environment for integrated models of biomolecular interaction networks. Genome Res 13: 2498-2504 
Singh AK, Kumar SR, Dwivedi V, Rai A, Pal S, Shasany AK, Nagegowda DA (2017) A WRKY transcription factor from Withania somnifera regulates triterpenoid withanolide accumulation and biotic stress tolerance through modulation of phytosterol and defense pathways. New Phytol 215: 1115-1131

Sun C, Wang J, Duan J, Zhao G, Weng X, Jia L (2017) Association of fruit and seed traits of Sapindus mukorossi germplasm with environmental factors in southern China. Forests 8

Tang QY, Geng C, Song WL, Wei F, Wei KH, He SM, Zhang G, Tang J, Li Y, Lin Y, Yang S (2019) Transcriptome analysis of Panax zingiberensis identifies genes encoding oleanolic acid glucuronosyltransferase involved in the biosynthesis of oleanane-type ginsenosides. Planta 249: 393-406

Tian F, Yang DC, Meng YQ, Jin JP, Gao G (2019) PlantRegMap: charting functional regulatory maps in plants. Nucleic Acids Research: gkz1020

Trapnell C, Williams BA, Pertea G, Mortazavi A, Kwan G, Van Baren MJ, Salzberg SL, Wold BJ, Pachter L (2010) Transcript assembly and quantification by RNA-Seq reveals unannotated transcripts and isoform switching during cell differentiation. Nature Biotechnology 28: 511-515

Umer MJ, Safdar LB, Gebremeskel H, Zhao S, Yuan P, Zhu H, Kaseb MO, Anees M, Lu X, He N, Gong C, Liu W (2020) Identification of key gene networks controlling organic acid and sugar metabolism during watermelon fruit development by integrating metabolic phenotypes and gene expression profiles. Hortic Res: 193

Upadhyay A, Singh DK (2012) Pharmacological effects of Sapindus mukorossi. Revista Do Instituto De Medicina Tropical De Sao Paulo 54: 273-280

Wang P, Wei Y, Fan Y, Liu Q, Wei W, Yang C, Zhang L, Zhao G, Yue J, Yan X, Zhou Z (2015) Production of bioactive ginsenosides $\mathrm{Rh} 2$ and $\operatorname{Rg} 3$ by metabolically engineered yeasts. Metab Eng 29

Wang R, Shu P, Zhang C, Zhang J, Chen Y, Zhang Y, Du K, Xie Y, Li M, Ma T, Zhang Y, Li Z, Grierson D, Pirrello J, Chen K, Bouzayen M, Zhang B, Liu M (2021) Integrative analyses of metabolome and genome-wide transcriptome reveal the regulatory network governing flavor formation in kiwifruit (Actinidia chinensis). New Phytol

Wei W, Wang P, Wei Y, Liu Q, Yang C, Zhao G, Yue J, Yan X, Zhou Z (2015) Characterization of Panax ginseng UDP-glycosyltransferases catalyzing protopanaxatriol and biosyntheses of bioactive ginsenosides F1 and Rh1 in metabolically engineered yeasts. Mol Plant 8: 1412-1424

Xu J, Wu S, Xu Y, Ge Z, Sui C, Wei J (2019) Overexpression of BcbZIP134 negatively regulates the biosynthesis of saikosaponins. Plant Cell Tiss Org 137: 297-308

Xu Y, Chen Z, Jia L, Weng X (2021) Advances in understanding of the biosynthetic pathway and regulatory mechanism of triterpenoid saponins in plants. Scientca Sinica Vitae 51: 525-555

Xu Y, Gao Y, Chen Z, Zhao G, Liu J, Wang X, Gao S, Zhang D, Jia L (2021) Metabolomics analysis of the soapberry (Sapindus mukorossi Gaertn.) pericarp during fruit development and ripening based on UHPLC-HRMS. Sci Rep 11: 11657

Xu Y, Jia L, Chen Z, Gao Y (2018) Advances on triterpenoid saponin of Sapindus mukorossi. Chemistry 81: 1078-1088

Yang JL, Hu ZF, Zhang TT, Gu AD, Gong T (2018) Progress on the studies of the key 
enzymes of ginsenoside biosynthesis. Molecules 23: 589

Yi D, Zhang H, Lai B, Liu L, Pan X, Ma Z, Wang Y, Xie J, Shi S, Wei Y (2020) Integrative analysis of the coloring mechanism of red longan pericarp through metabolome and transcriptome analyses. J Agr Food Chem 69 Dai M (2021) Genomic basis underlying the metabolome-mediated drought adaptation of maize. Genome Biol 22: 260

Zhang H, Shibuya M, Yokota S, Ebizuka Y (2003) Oxidosqualene cyclases from cell suspension cultures of Betula platyphylla var. japonica: molecular evolution of oxidosqualene cyclases in higher plants. Biol Pharm Bull: 642-650

Zhao G, Gao Y, Gao S, Xu Y, Liu J, Sun C, Gao Y, Liu S, Chen Z, Jia L (2019) The phenological growth stages of Sapindus mukorossi according to BBCH scale. Forests 10: 462

Zhao Y, Li C (2018) Biosynthesis of plant triterpenoid saponins in microbial cell factories. J Agr Food Chem 66: 12155-12165 
A

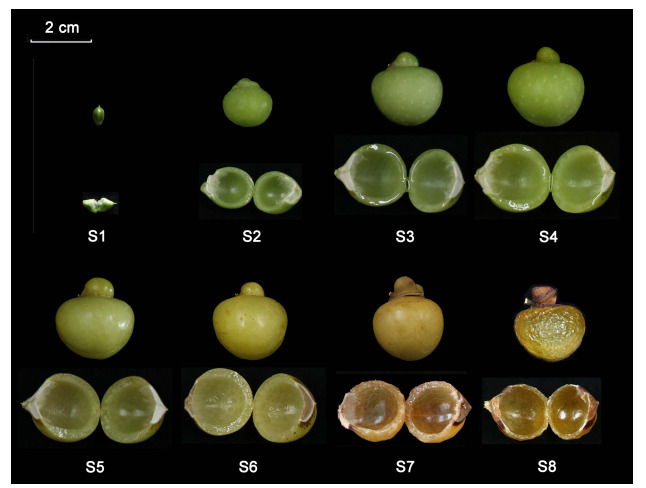

C

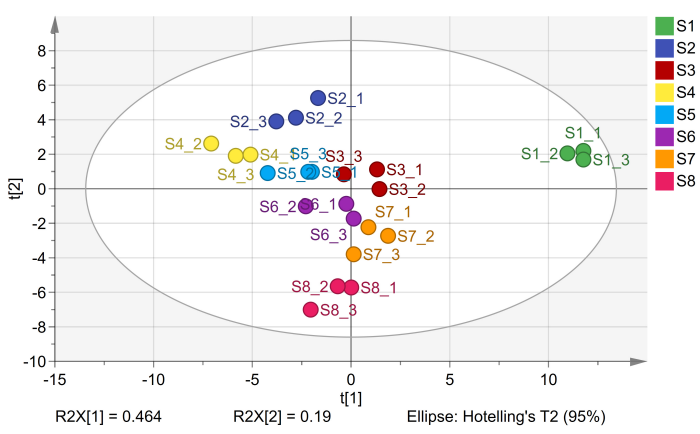

B

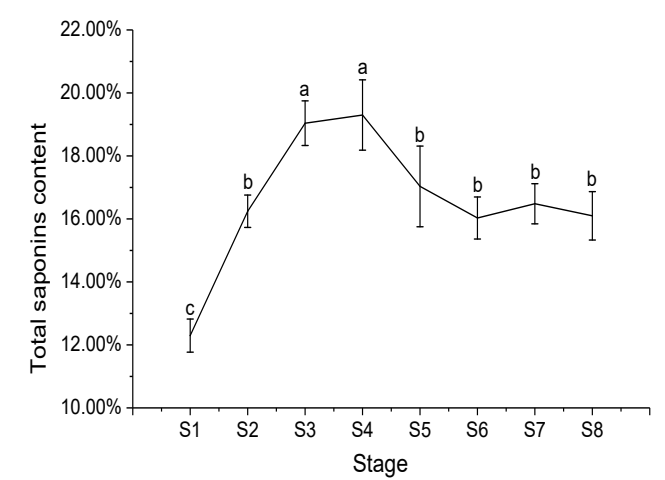

D

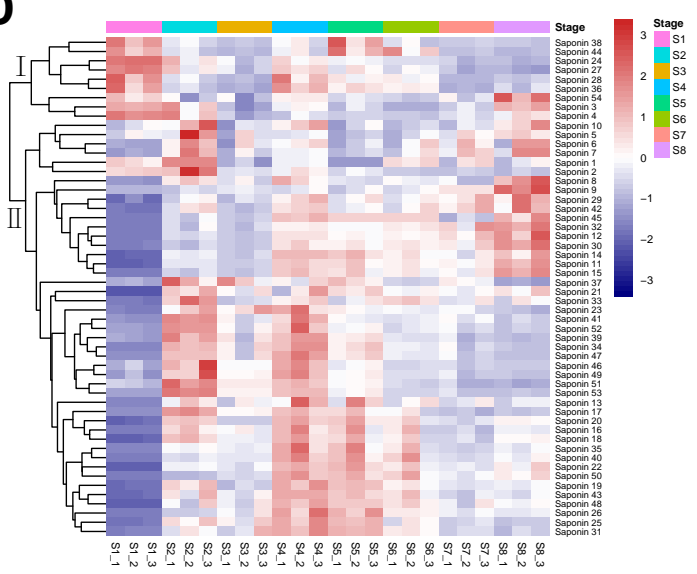

E

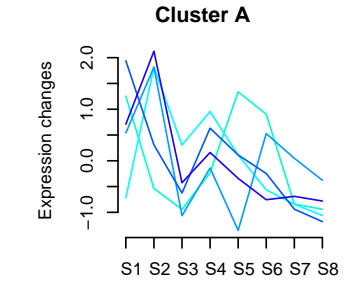

Time

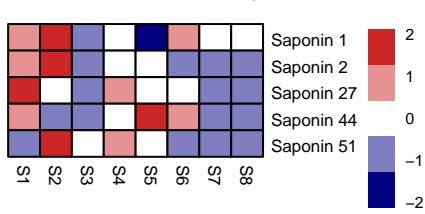

Cluster B

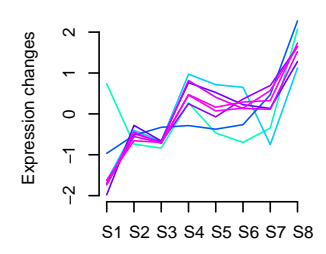

Time

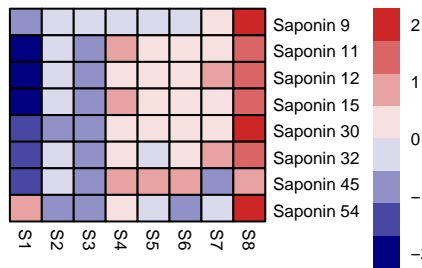

Cluster C

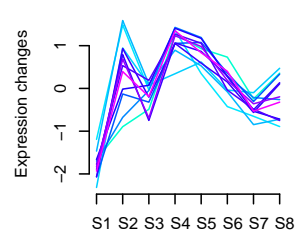

Time

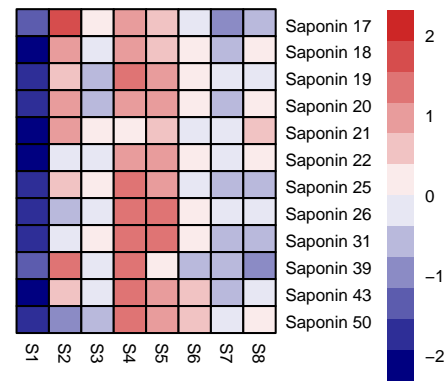

Figure 1. Images of soapberry fruits and dynamic changes of saponins in the pericarp at eight growth stages. (A) Images of soapberry fruits and pericarps at eight growth stages. (B) Dynamic changes of total saponin contents of soapberry pericarp at eight growth stages. Means $\pm \mathrm{SD}$ $(\mathrm{n}=3)$. Means in different letters $(\mathrm{a}-\mathrm{b})$ are significantly different at $P<0.05$ by ANOVA on Duncan's multiple range test. (C) Principal component analysis (PCA) of detected saponins. (D) Hierarchical clustering heat map of detected saponins. (e) Upper: Temporal expression patterns of DASs. All DASs were classified into three clusters based on their contents using the Mfuzz R package. (E) Lower: Heatmap of DASs accumulation level in cluster A, cluster B and cluster C. 


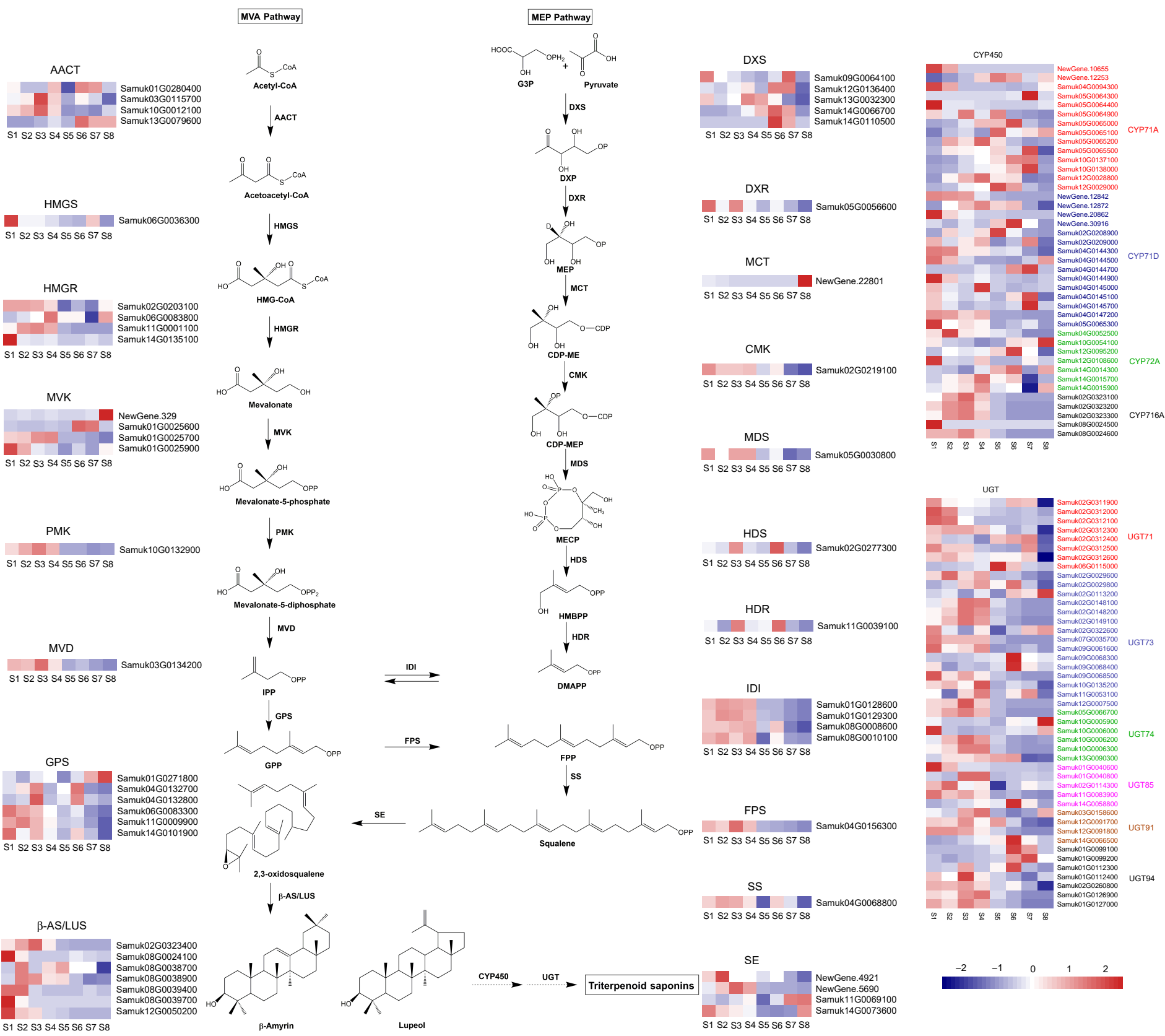

Figure 2. Proposed pathways for triterpenoid saponin biosynthesis in soapberry. Expression levels of genes encoding enzymes that catalyze each step of the triterpenoid saponin biosynthesis pathway are shown. The higher and lower expression level of genes are indicated in firebrick and navy, respectively. 
A

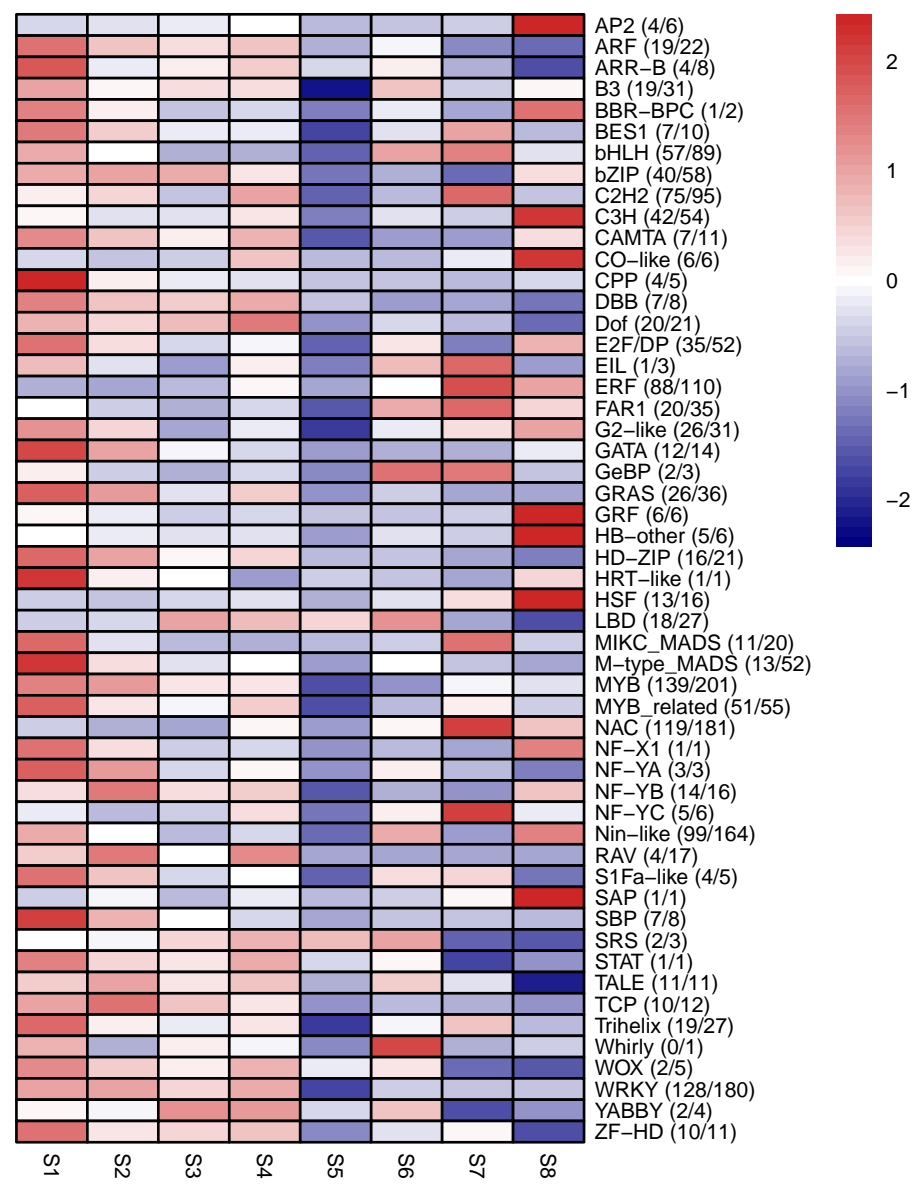

B
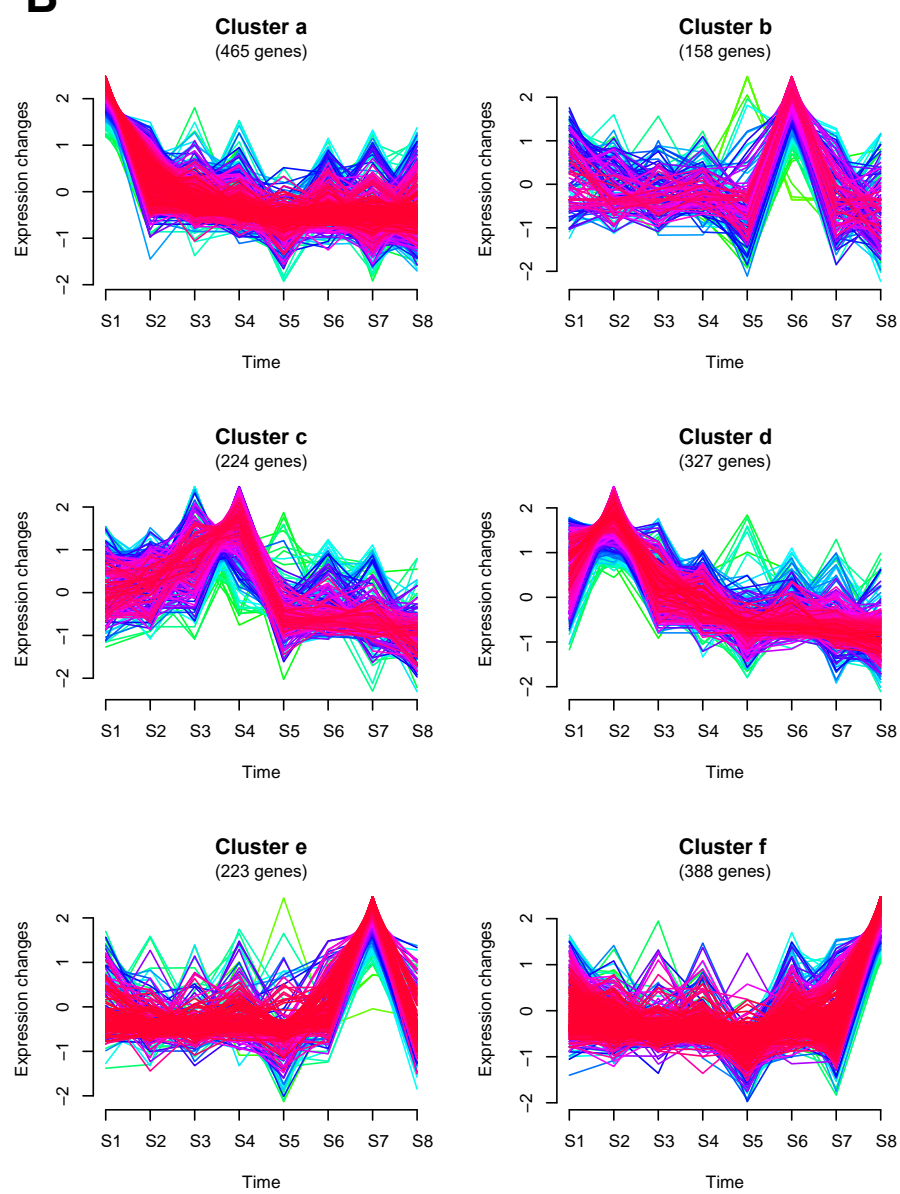

Figure 3. Expression dynamics of TF families. (A) The colour shows the total FPKM of all TFs of a particular TF family. The first number in parentheses is the number of DEGs in a TF family, and the second number refers to the total number of genes in that TF family as annotated in PlantTFDB. (B) Expression profiles of all TFs by Mfuzz clustering analysis. 

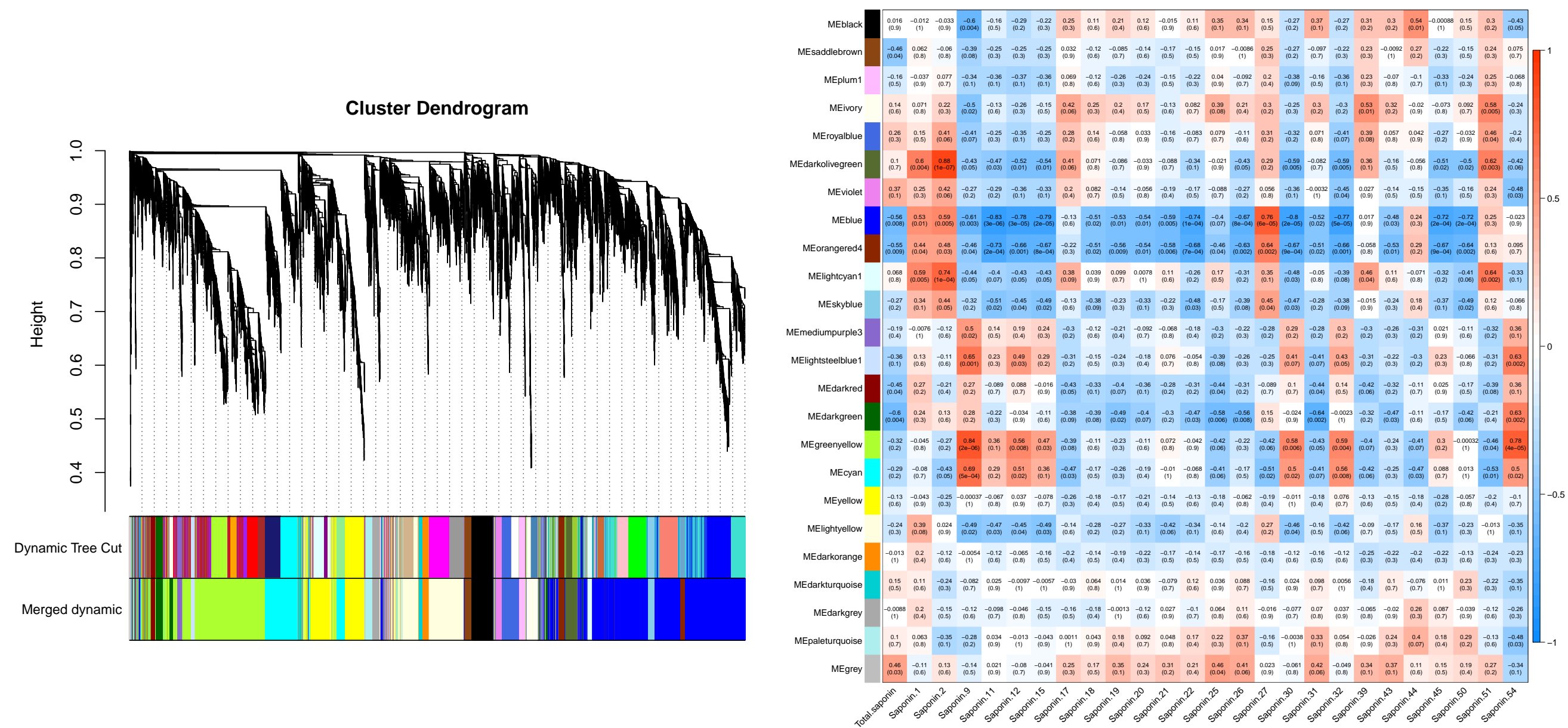

C

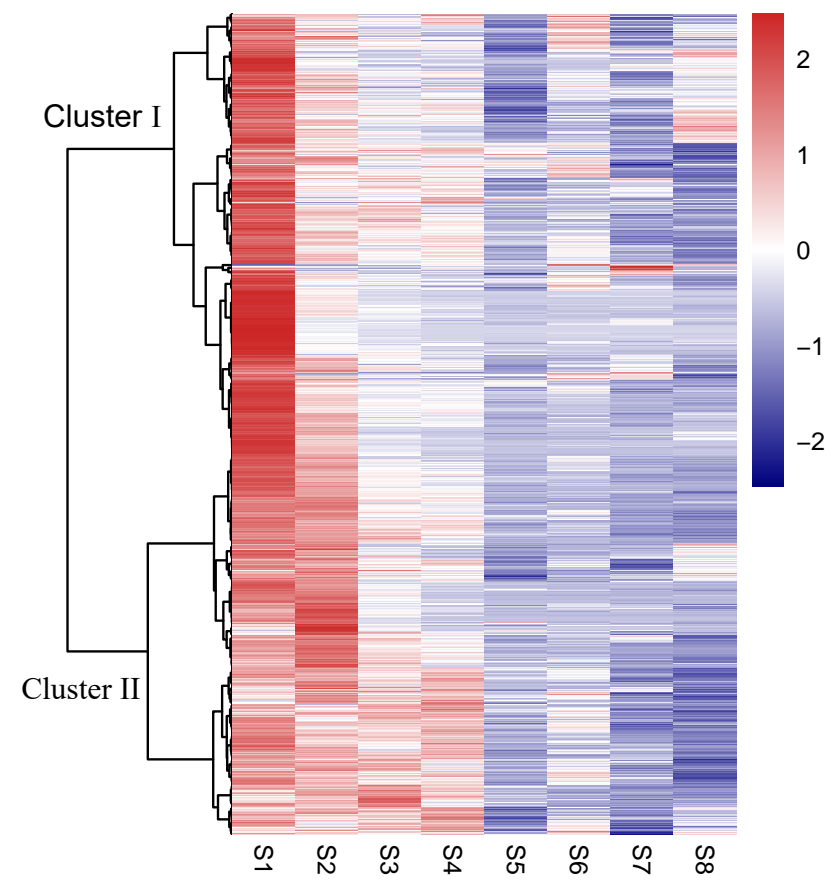

D
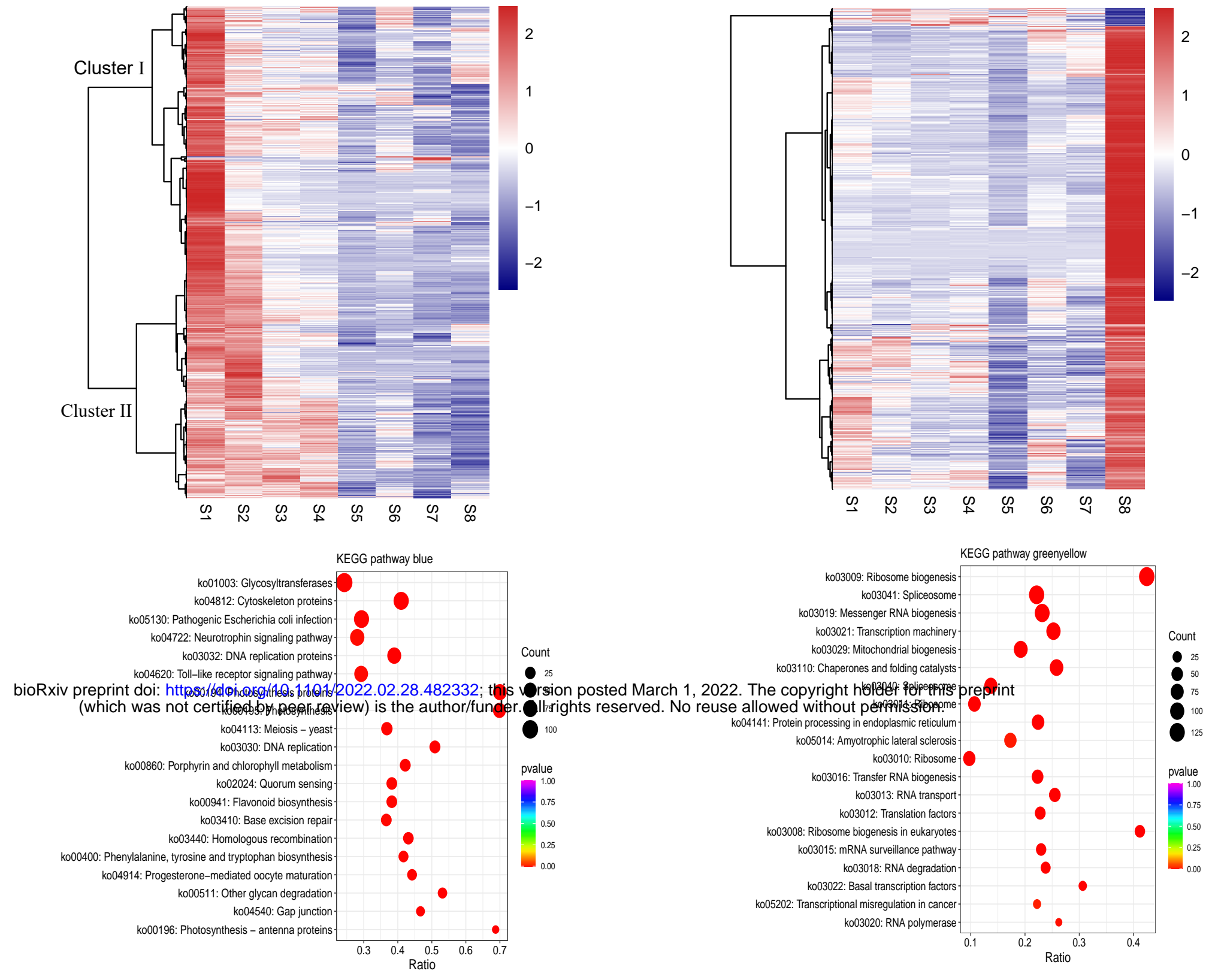

Figure 4. WGCNA of all expressed genes. (A) Hierarchical clustering tree (cluster dendrogram) showing 24 modules of co-expressed genes by WGCNA. Each leaf of tree corresponds to one gene. The major tree branches constitute 24 modules, labeled with different colors. (B) Module-saponin relationship. Each row represents a module. Each column represents a specific saponin (detailed information reference Table S4). The value in each cell at the rowcolumn intersection represents the correlation coefficient between the module and the saponin and is displayed according to the color scale on the right. The value in parentheses in each cell represents the P value. (C) Upper: heat map of genes in the blue module; Lower: the KEGG enrichment analysis of the genes in the blue module showed only the top 20 pathways with the most significant enrichment. (D) Upper: heat map of genes in the greenyellow module; Lower: the KEGG enrichment analysis of the genes in the greenyellow module showed only the top 20 pathways with the most significant enrichment. 


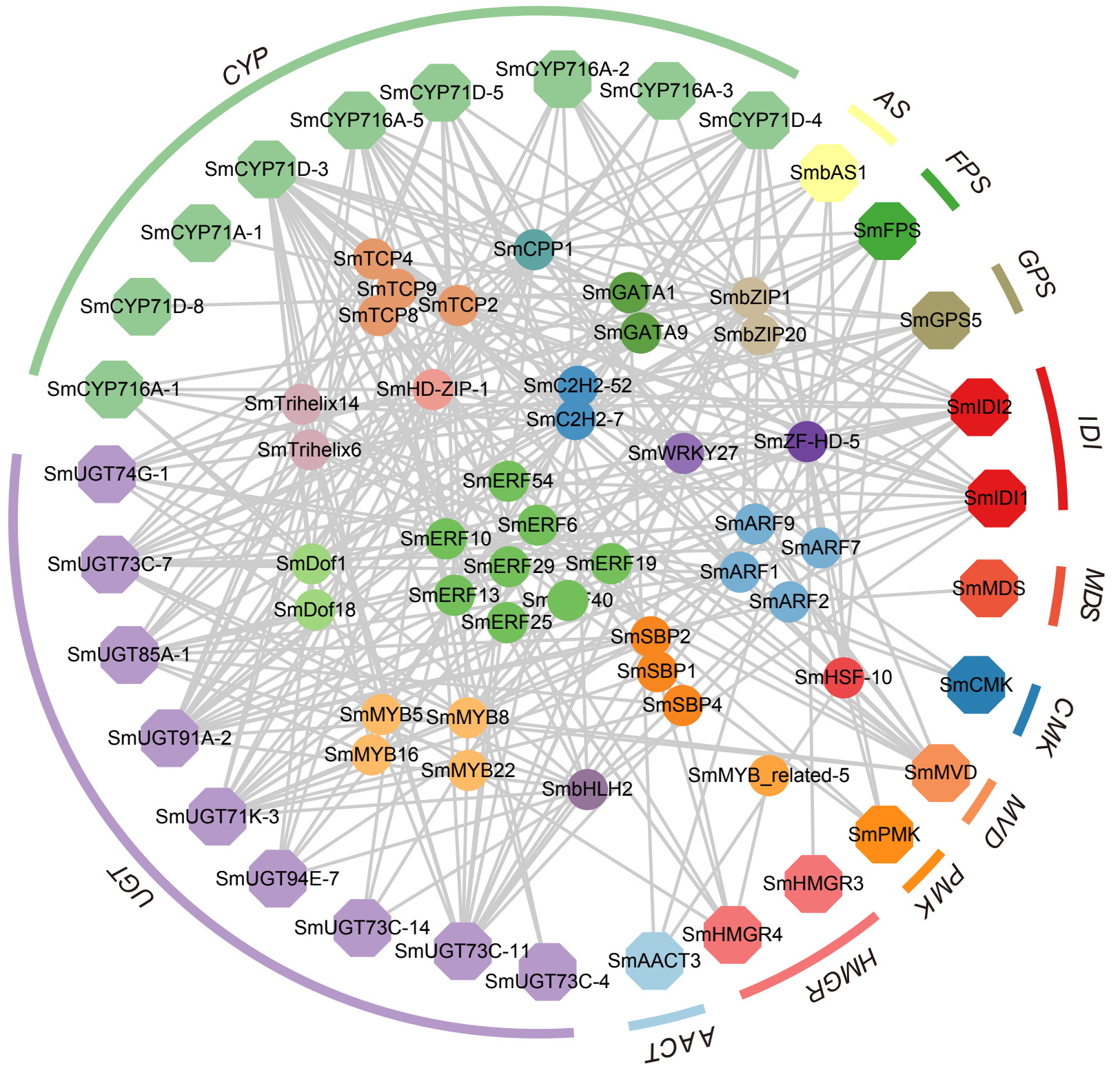

Figure 5. The regulatory network of triterpenoid saponin biosynthesis in soapberry. Hexagon with different colors indicate different families of structural genes associated with saponin biosynthesis in the blue module. Circles with different colors indicate different families of TFs characterize in the same module which transcripts are highly correlated with expression of structural genes. 
bioRxiv preprint doi: https://doi.org/10.1101/2022.02.28.482332; this version posted March 1,2022 . The copyright holder for this preprint (which was not certified by peer review) is the author/funder. All rights reserved. No reuse allowed without permission.
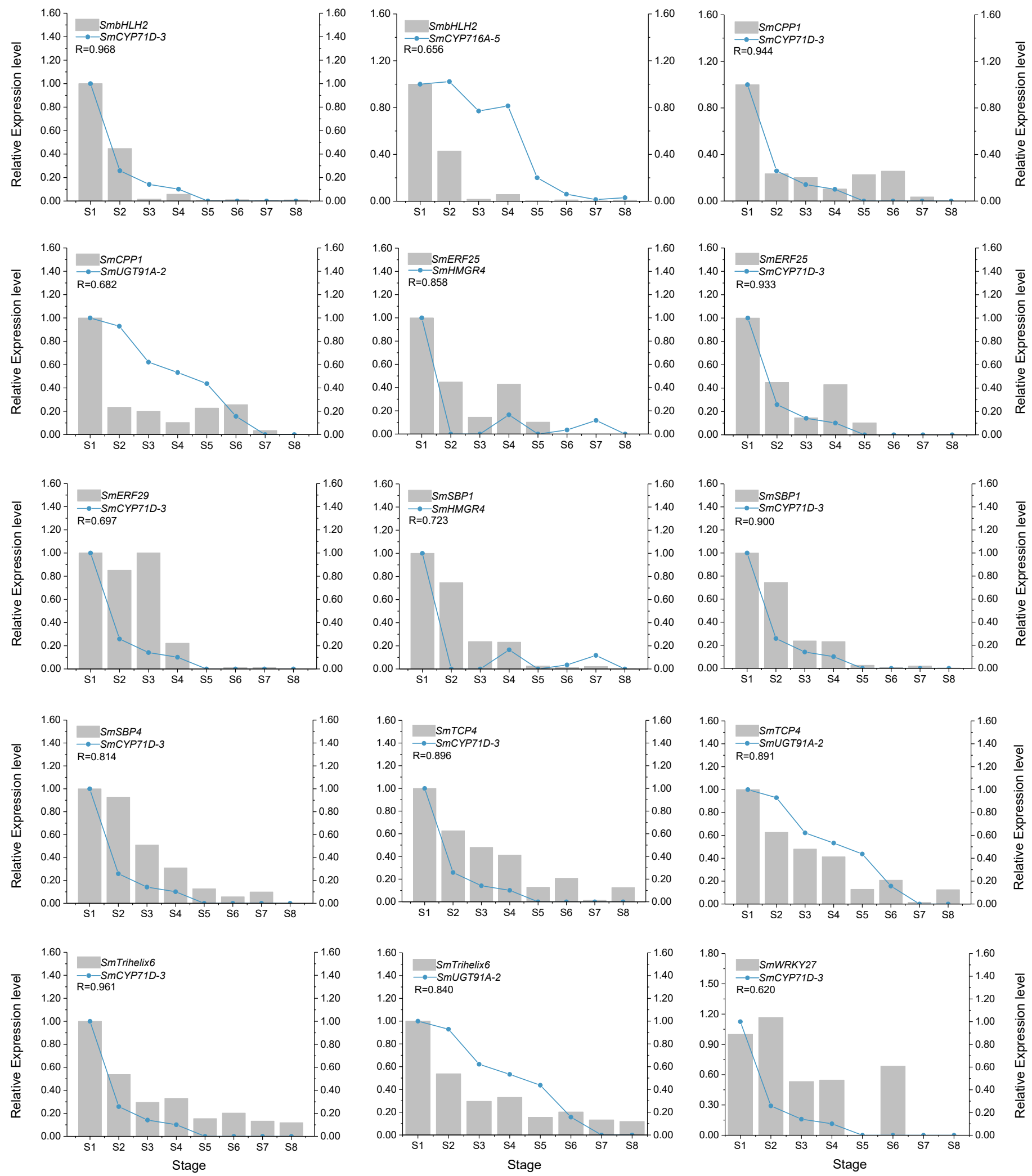

Figure 6. Expression correlation of TFs and their predicted target genes. The bars and lines indicate the relative expression level of TFs and their corresponding target genes in the eight growth stages of soapberry pericarps. The Y-axis on the left and right represents the abundance of the TFs and their target genes, respectively. SmACT was used for normalizing the relative expression of these encoding genes. The expression level of the genes in the stage $\mathrm{S} 1$ were set as 1.0. Relative expression level was calculated using the $2^{-\Delta \Delta C t}$ method. Data indicate the mean values of three biological replications. 
A

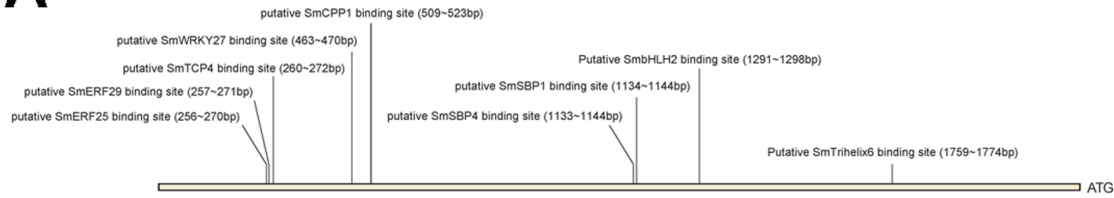

pGADT7+ProSmCYP71D-3
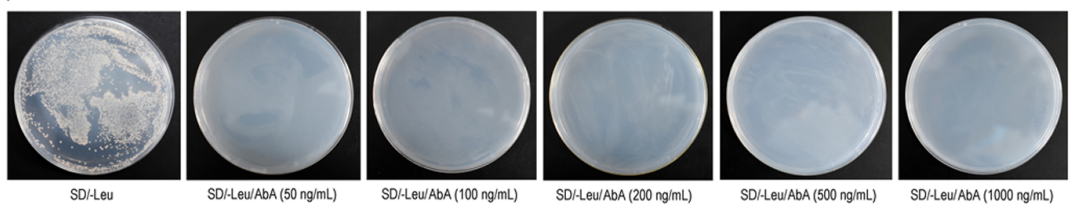

SD/-Leu

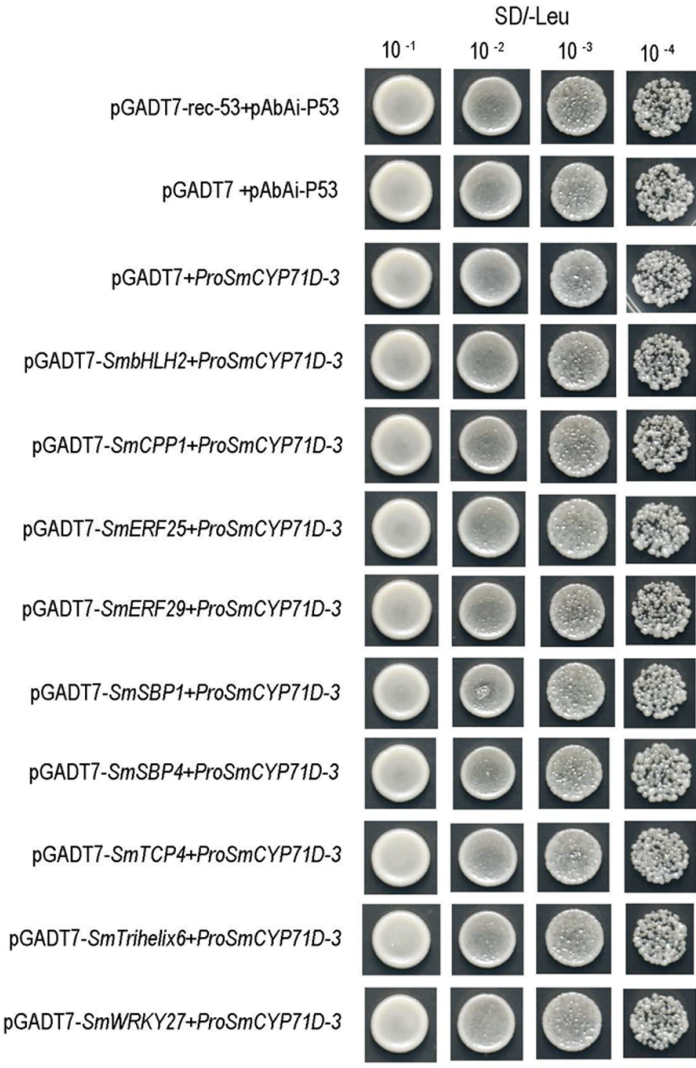

B

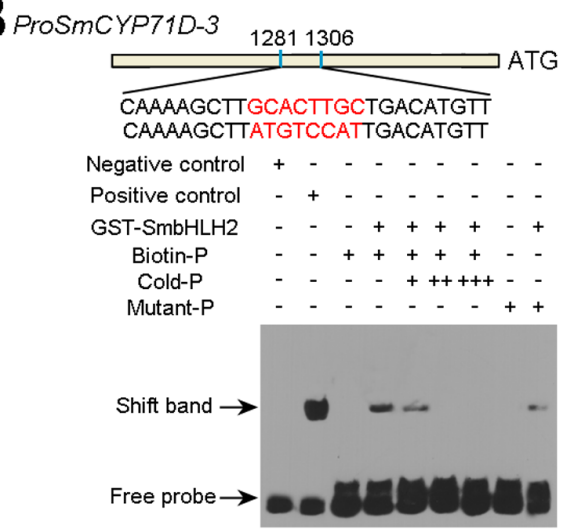

ProSmCYP71D-3 253278

$\widehat{\text { AAGAAGTGGCGGCCCCAAACAATAGT }}$ AAGAAGCAATAATTTGTGGCAATAGT Negative control $+--_{-}--_{-}-$ Positive control $-+{ }_{-}--_{-}-{ }_{-}$

GST-SmTCP4 - - - + + + + +

Biotin-P $-\quad+++++-$

Cold-P $\quad-\quad-\quad-\quad++++++-$

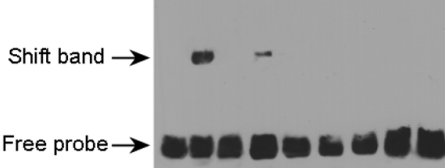

ProSmCYP71D-3

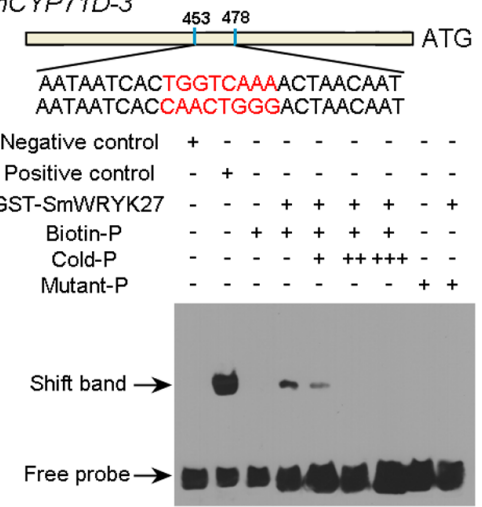

Figure 7. Identification of SmbHLH2, SmTCP4, and SmWRKY27 as key TFs regulating SmCYP7ID-3 in soapberry. (A) Upper: schematic illustration of the potential recognition elements of the nine TFs in the $2 \mathrm{~kb}$ promoter region of SmCYP71D-3. (A) Middle: no basal activities of ProSmCYP71D-3 were observed in yeast grown on SD medium lacking Leu in the presence of 50,100, 200, 500, and $1000 \mathrm{ng} / \mathrm{mL}-1 \mathrm{AbA}(\mathrm{SD} / \mathrm{Leu} / \mathrm{AbA})$. (A) Lower: yeast growth assays after Y1H reporter strains were transformed with effector containing the ORF of different TFs or empty (negative control). Interaction was determined based on the ability of transformed yeast to grow on SD medium lacking Leu in the presence of $50 \mathrm{ng} / \mathrm{mL}-1$ (SD/-Leu/AbA). (B) SmbHLH2, SmTCP4, and SmWRKY27 binding to the promoter of SmCYP71D-3. The WT probe containing the corresponding TF binding site was biotin-l abeled. Competition for TF binding was performed with cold probes. The symbols - and + represent absence or presence of the probes and GST-tagged TF protein. The core binding sequences are highlighted red; The upper sequence is the prediction TF binding site sequence, and the lower sequence is the mutation probe sequence. 


\section{Parsed Citations}

Anders S, Huber W (2010) Differential expression analysis for sequence count data. Genome Biology 11: R106 Google Scholar: Author Only Title Only Author and Title

Augustin JM, Kuzina V, Andersen SB, Bak S (2011) Molecular activities, biosynthesis and evolution of triterpenoid saponins. Phytochemistry 72: 435-457

Google Scholar: Author Only Title Only Author and Title

Dai L, Liu C, Zhu Y, Zhang J, Men Y, Zeng Y, Sun Y (2015) Functional characterization of cucurbitadienol synthase and triterpene glycosyltransferase involved in biosynthesis of mogrosides from Siraitia grosvenorii. Plant Cell Physiol 56: 1172-1182

Google Scholar: Author Only Title Only Author and Title

Deng B, Huang Z, Ge F, Liu D, Lu R, Chen CJJoPGR (2017) An AP2/ERF family transcription factor PnERF1 raised the biosynthesis of saponins in Panax notoginseng. J Plant Growth Regul: 691-701

Google Scholar: Author Only Title Only Author and Title

Erthmann PØ, Agerbirk N, Bak S (2018) A tandem array of UDP-glycosyltransferases from the UGT73C subfamily glycosylate sapogenins, forming a spectrum of monoand bisdesmosidic saponins. Plant Mol Biol 97: 37-55

Google Scholar: Author Only Title Only Author and Title

Fiallos-Jurado J, Pollier J, Moses T, Arendt P, Barriga-Medina N, Morillo E, Arahana V, Torres M, Goossens A, Leon-Reyes A (2016) Saponin determination, expression analysis and functional characterization of saponin biosynthetic genes in

Chenopodium quinoa leaves. Plant Sci 250: 188-197

Google Scholar: Author Only Title Only Author and Title

Ghosh S (2017) Triterpene structural diversification by plant cytochrome P450 enzymes. Front Plant Sci 8: 1886 Google Scholar: Author Only Title Only Author and Title

Grant CE, Bailey TL, Noble WS (2011) FIMO: Scanning for occurrences of a given motif. Bioinformatics 27: 1017-1018 Google Scholar: Author Only Title Only Author and Title

Han JY, Chun JH, Oh SA, Park SB, Hwang HS, Lee H, Choi YE (2017) Transcriptomic analysis of Kalopanax septemlobus and characterization of KsBAS, CYP716A94, and CYP72A397 genes involved in hederagenin saponin biosynthesis. Plant Cell Physiol 59: 319-330

Google Scholar: Author Only Title Only Author and Title

Haralampidis K, Trojanowska M, Osbourn AE (2002) Biosynthesis of triterpenoid saponins in plants. Adv Biochem Eng Biot 75: 31 Google Scholar: Author Only Title Only Author and Title

Hayashi H, Huang P, Kirakosyan A, Inoue K, Hiraoka N, Ikeshiro Y, Kushiro T, Shibuya M, Ebizuka Y (2001) Cloning and characterization of a cDNAencoding $\beta$-amyrin synthase involved in glycyrrhizin and soyasaponin biosyntheses in licorice. Biol Pharm Bull 24: 912-916

Google Scholar: Author Only Title Only Author and Title

Hayashi H, Huang P, Takada S, Obinata M, Inoue K, Shibuya M, Ebizuka Y (2004) Differential expression of three oxidosqualene cyclase mRNAs in Glycyrrhiza glabra. Biol Pharm Bull 27: 1086-1092

Google Scholar: Author Only Title Only Author and Title

Hellman LM, Fried MG (2007) Electrophoretic mobility shift assay (EMSA) for detecting protein-nucleic acid interactions. Nat Protoc 2: 1849-1861

Google Scholar: Author Only Title Only Author and Title

Jia L, Sun C (2012) Research progress of biodiesel tree Sapindus mukorossi. J. China Agric. Univ. 17: 191-196

Google Scholar: Author Only Title Only Author and Title

Kim D, Langmead B, Salzberg SL (2015) HISAT: a fast spliced aligner with low memory requirements. Nature Methods 12: 357-360 Google Scholar: Author Only Title Only Author and Title

Krokida A, Delis C, Geisler K, Garagounis C, Tsikou D, Peña-Rodríguez L, Katsarou D, Field B, Osbourn AE, Papadopoulou KK (2013) A metabolic gene cluster in Lotus japonicus discloses novel enzyme functions and products in triterpene biosynthesis.

New Phytol 200: 675-690

Google Scholar: Author Only Title Only Author and Title

Langfelder P, Horvath S (2008) WGCNA: an R package for weighted correlation network analysis. BMC Bioinformatics 9: 559-559 Google Scholar: Author Only Title Only Author and Title

Li S (1975) Compendium of Materia Medica. People's Medical Publishing House, Beijing

Google Scholar: Author Only Title Only Author and Title 
Li Y, Chen Y, Zhou L, You S, Deng H, Chen Y, Aseekh S, Yuan Y, Fu R, Zhang Z, Su D, Fernie AR, Bouzayen M, Ma T, Liu M, Zhang Y (2020) MicroTom metabolic network: rewiring tomato metabolic regulatory network throughout the growth cycle. Mol Plant 13: 1203-1218

Google Scholar: Author Only Title Only Author and Title

Liu Y, Lv J, Liu Z, Wang J, Yang B, Chen W, Ou L, Dai X, Zhang Z, Zou X (2020) Integrative analysis of metabolome and transcriptome reveals the mechanism of color formation in pepper fruit (Capsicum annuum L.). Food Chem $306: 125629$ Google Scholar: Author Only Title Only Author and Title

Love MI, Huber W, Anders S (2014) Moderated estimation of fold change and dispersion for RNA-seq data with DESeq2. Genome Biology 15: 550

Google Scholar: Author Only Title Only Author and Title

Lu C, Zhao S, Wang X (2017) Functional regulation of a UDP-glucosyltransferase gene (Pq3-O-UGT1) by RNAinterference and overexpression in Panax quinquefolius. Plant Cell Tiss Organ Cult 129: 445-456

Google Scholar: Author Only Title Only Author and Title

Lu C, Zhao S, Wei G, Zhao H, Qu Q (2017) Functional regulation of ginsenoside biosynthesis by RNA interferences of a UDP. glycosyltransferase gene in Panax ginseng and Panax quinquefolius. Plant Physiol Bioch 111: 67-76

Google Scholar: Author Only Title Only Author and Title

Meng J, Wang B, He G, Wang Y, Tang X, Wang S, Ma Y, Fu C, Chai G, Zhou G (2019) Metabolomics integrated with transcriptomics reveals redirection of the phenylpropanoids metabolic flux in Ginkgo biloba. J Agr Food Chem 67: 3284-3291

Google Scholar: Author Only Title Only Author and Title

Mertens J, Pollier J, Bossche RV, Lopez-Vidriero I, Franco-Zorrilla JM, Goossens A(2016) The bHLH transcription factors TSAR1 and TSAR2 regulate triterpene saponin biosynthesis in Medicago truncatula. Plant Physiol 170: $194-210$

Google Scholar: Author Only Title Only Author and Title

Miettinen K, Pollier J, Buyst D, Arendt P, Csuk R, Sommerwerk S, Moses T, Mertens J, Sonawane PD, Pauwels L, Aharoni A, Martins J, Nelson DR, Goossens A(2017) The ancient CYP716 family is a major contributor to the diversification of eudicot triterpenoid biosynthesis. Nat Commun 8: 14153

Google Scholar: Author Only Title Only Author and Title

Mou W, Li D, Luo Z, Li L, Mao L, Ying T (2018) SIAREB1 transcriptional activation of NOR is involved in abscisic acid-modulated ethylene biosynthesis during tomato fruit ripening. Plant Sci 276: 239-249

Google Scholar: Author Only Title Only Author and Title

Ndamba J, Lemmich E, Mølgaard P (1994) Investigation of the diurnal, ontogenetic and seasonal variation in the molluscicidal saponin content of Phytolacca dodecandra aqueous berry extracts. Phytochemistry 35: 95-99

Google Scholar: Author Only Title Only Author and Title

Nett RS, Lau W, Sattely ES (2020) Discovery and engineering of colchicine alkaloid biosynthesis. Nature 584: 148-153 Google Scholar: Author Only Title Only Author and Title

Ni J, Zhao Y, Tao R, Yin L, Bai S (2020) Ethylene mediates the branching of the jasmonate- induced flavonoid biosynthesis pathway by suppressing anthocyanin biosynthesis in red Chinese pear fruits. Plant Biotechnol J 18: $1223-1240$

Google Scholar: Author Only Title Only Author and Title

Pertea M, Pertea G, Antonescu C, Chang TC, Mendell JT, Salzberg SL (2015) StringTie enables improved reconstruction of a transcriptome from RNAseq reads. Nature Biotechnology 33: 290-295

Google Scholar: Author Only Title Only Author and Title

Rahimi S, Kim J, Mijakovic I, Jung K, Choi G, Kim SC, Kim YJ (2019) Triterpenoid-biosynthetic udp-glycosyltransferases from plants. Biotechnol Adv 37: 107394

Google Scholar: Author Only Title Only Author and Title

Sawai S, Shindo T, Sato S, Kaneko T, Tabata S, Ayabe SI, Aoki T (2006) Functional and structural analysis of genes encoding oxidosqualene cyclases of Lotus japonicus. Plant Sci 170: 247-257

Google Scholar: Author Only Title Only Author and Title

Schramek N, Huber C, Schmidt S, Dvorski S, Kmispel N, Ostrozhenkova E (2014) Biosynthesis of ginsenosides in field-grown Panax ginseng. JSM Biotechnol Bioeng: 1033

Google Scholar: Author Only Title Only Author and Title

Shannon P, Markiel A, Ozier O, Baliga NS, Wang JT, Ramage D, Amin N, Schwikowski B, Ideker T (2003) Cytoscape: a software environment for integrated models of biomolecular interaction networks. Genome Res 13: 2498-2504

Google Scholar: Author Only Title Only Author and Title

Singh AK, Kumar SR, Dwivedi V, Rai A, Pal S, Shasany AK, Nagegowda DA(2017) AWRKY transcription factor from Withania 
somnifera regulates triterpenoid withanolide accumulation and biotic stress tolerance through modulation of phytosterol and defense pathways. New Phytol 215: 1115-1131

Google Scholar: Author Only Title Only Author and Title

Sun C, Wang J, Duan J, Zhao G, Weng X, Jia L (2017) Association of fruit and seed traits of Sapindus mukorossi germplasm with environmental factors in southern China. Forests 8

Google Scholar: Author Only Title Only Author and Title

Tang QY, Geng C, Song WL, Wei F, Wei KH, He SM, Zhang G, Tang J, Li Y, Lin Y, Yang S (2019) Transcriptome analysis of Panax zingiberensis identifies genes encoding oleanolic acid glucuronosyltransferase involved in the biosynthesis of oleanane-type ginsenosides. Planta 249: 393-406

Google Scholar: Author Only Title Only Author and Title

Tian F, Yang DC, Meng YQ, Jin JP, Gao G (2019) PlantRegMap: charting functional regulatory maps in plants. Nucleic Acids

Research: gkz1020

Google Scholar: Author Only Title Only Author and Title

Trapnell C, Williams BA, Pertea G, Mortazavi A, Kwan G, Van Baren MJ, Salzberg SL, Wold BJ, Pachter L (2010) Transcript assembly and quantification by RNASeq reveals unannotated transcripts and isoform switching during cell differentiation. Nature Biotechnology 28: 511-515

Google Scholar: Author Only Title Only Author and Title

Umer MJ, Safdar LB, Gebremeskel H, Zhao S, Yuan P, Zhu H, Kaseb MO, Anees M, Lu X, He N, Gong C, Liu W(2020) Identification of key gene networks controlling organic acid and sugar metabolism during watermelon fruit development by integrating metabolic phenotypes and gene expression profiles. Hortic Res: 193

Google Scholar: Author Only Title Only Author and Title

Upadhyay A Singh DK (2012) Pharmacological effects of Sapindus mukorossi. Revista Do Instituto De Medicina Tropical De Sao Paulo 54: 273-280

Google Scholar: Author Only Title Only Author and Title

Wang P, Wei Y, Fan Y, Liu Q, Wei W, Yang C, Zhang L, Zhao G, Yue J, Yan X, Zhou Z(2015) Production of bioactive ginsenosides Rh2 and Rg3 by metabolically engineered yeasts. Metab Eng 29

Google Scholar: Author Only Title Only Author and Title

Wang R, Shu P, Zhang C, Zhang J, Chen Y, Zhang Y, Du K, Xie Y, Li M, Ma T, Zhang Y, Li Z, Grierson D, Pirrello J, Chen K, Bouzayen M, Zhang B, Liu M (2021) Integrative analyses of metabolome and genome-wide transcriptome reveal the regulatory network governing flavor formation in kiwifruit (Actinidia chinensis). New Phytol

Google Scholar: Author Only Title Only Author and Title

Wei W, Wang P, Wei Y, Liu Q, Yang C, Zhao G, Yue J, Yan X, Zhou Z(2015) Characterization of Panax ginseng UDPglycosyltransferases catalyzing protopanaxatriol and biosyntheses of bioactive ginsenosides F1 and Rh1 in metabolically engineered yeasts. Mol Plant 8: 1412-1424

Google Scholar: Author Only Title Only Author and Title

Xu J, Wu S, Xu Y, Ge Z, Sui C, Wei J (2019) Overexpression of BcbZP134 negatively regulates the biosynthesis of saikosaponins. Plant Cell Tiss Org 137: 297-308

Google Scholar: Author Only Title Only Author and Title

Xu Y, Chen Z, Jia L, Weng X (2021) Advances in understanding of the biosynthetic pathway and regulatory mechanism of triterpenoid saponins in plants. Scientca Sinica Vitae 51: 525-555

Google Scholar: Author Only Title Only Author and Title

Xu Y, Gao Y, Chen Z, Zhao G, Liu J, Wang X, Gao S, Zhang D, Jia L (2021) Metabolomics analysis of the soapberry (Sapindus mukorossi Gaertn.) pericarp during fruit development and ripening based on UHPLC-HRMS. Sci Rep 11: 11657

Google Scholar: Author Only Title Only Author and Title

Xu Y, Jia L, Chen Z, Gao Y (2018) Advances on triterpenoid saponin of Sapindus mukorossi. Chemistry 81: 1078-1088

Google Scholar: Author Only Title Only Author and Title

Yang JL, Hu ZF, Zhang TT, Gu AD, Gong T (2018) Progress on the studies of the key enzymes of ginsenoside biosynthesis.

Molecules 23: 589

Google Scholar: Author Only Title Only Author and Title

Yi D, Zhang H, Lai B, Liu L, Pan X, Ma Z, Wang Y, Xie J, Shi S, Wei Y (2020) Integrative analysis of the coloring mechanism of red longan pericarp through metabolome and transcriptome analyses. J Agr Food Chem 69

Google Scholar: Author Only Title Only Author and Title

Zhang F, Wu J, Sade N, Wu S, Egbaria A, Fernie AR, Yan J, Qin F, Chen W, Brotman Y, Dai M (2021) Genomic basis underlying the metabolome-mediated drought adaptation of maize. Genome Biol 22: 260 
Zhang H, Shibuya M, Yokota S, Ebizuka Y (2003) Oxidosqualene cyclases from cell suspension cultures of Betula platyphylla var. japonica: molecular evolution of oxidosqualene cyclases in higher plants. Biol Pharm Bull: 642-650

Google Scholar: Author Only Title Only Author and Title

Zhao G, Gao Y, Gao S, Xu Y, Liu J, Sun C, Gao Y, Liu S, Chen Z, Jia L (2019) The phenological growth stages of Sapindus mukorossi according to BBCH scale. Forests 10: 462

Google Scholar: Author Only Title Only Author and Title

Zhao Y, Li C (2018) Biosynthesis of plant triterpenoid saponins in microbial cell factories. J Agr Food Chem 66: 12155-12165

Google Scholar: Author Only Title Only Author and Title 